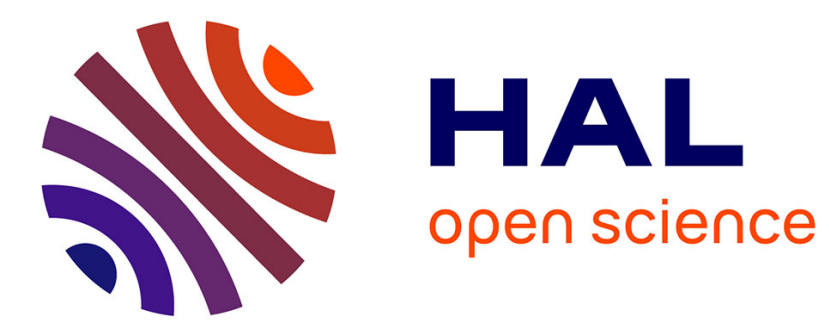

\title{
Pyramidal Adaptive Meshing for Digital Image Correlation Dealing With Cracks
}

\author{
V F Sciuti, R Vargas, R B Canto, François Hild
}

\section{To cite this version:}

V F Sciuti, R Vargas, R B Canto, François Hild. Pyramidal Adaptive Meshing for Digital Image Correlation Dealing With Cracks. Engineering Fracture Mechanics, 2021, 256, pp.107931. 10.1016/j.engfracmech.2021.107931 . hal-03310448

\section{HAL Id: hal-03310448 \\ https://hal.science/hal-03310448}

Submitted on 30 Jul 2021

HAL is a multi-disciplinary open access archive for the deposit and dissemination of scientific research documents, whether they are published or not. The documents may come from teaching and research institutions in France or abroad, or from public or private research centers.
L'archive ouverte pluridisciplinaire HAL, est destinée au dépôt et à la diffusion de documents scientifiques de niveau recherche, publiés ou non, émanant des établissements d'enseignement et de recherche français ou étrangers, des laboratoires publics ou privés. 


\title{
Pyramidal Adaptive Meshing for Digital Image Correlation Dealing With Cracks
}

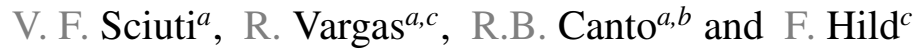 \\ ${ }^{a}$ Federal University of São Carlos, Graduate Program in Materials Science and Engineering (PPGCEM), 13565-905, São Carlos-SP, Brazil \\ ${ }^{b}$ Federal University of São Carlos (UFSCar), Department of Materials Engineering (DEMa), 13565-905, São Carlos-SP, Brazil, Brazil \\ ${ }^{c}$ Université Paris-Saclay, ENS Paris-Saclay, CNRS, LMT - Laboratoire de Mécanique et Technologie, 91190 Gif-sur-Yvette, France
}

\section{ARTICLE INFO}

Keywords:

crack

Lagrange multipliers

artificial images

wedge splitting test

$\mathrm{MgO}$ hydration crack network

computational cost

\begin{abstract}
A B S TR ACT
8

A mesh refinement procedure designed for Digital Image Correlation analyses of cracked media is proposed, based on the pixel color for an artificial case, and the crack opening displacement field for two experimental cases. Although evidence that this procedure can improve every case if tiny elements are sought, it is also shown that the size of matrices and consequently, the computational cost can be reduced while maintaining accuracy, especially for low surface crack densities (e.g., single macrocrack).
\end{abstract}

\footnotetext{
*Corresponding author

vfsciuti@gmail.com (V.F. Sciuti)

ORCID(s): 0000-0002-3709-3189 (V.F. Sciuti); 0000-0002-5524-1886 (R. Vargas); 0000-0002-9286-9912 (R.B. Canto);
}

0000-0001-5553-0066 (F. Hild) 


\section{Introduction}

Many materials remain functional with cracks before complete failure. Not only predicting such damage to avoid accidents but also its monitoring is a constant field of development. Since the introduction of Digital Image Correlation (DIC) in the early 1980s [1, 2], it has seen many developments [3]. Among all applications of this technique [4, 5, 6], the analysis of cracked bodies has received a lot of attention since the early days of DIC [7, 8]. In the field of fracture mechanics, it was shown that stress intensity factors [8,9], energy release rates [10], parameters of cohesive zone models $[11,12,13]$ could be determined via comparisons of measured displacements with simulations or available closed-form solutions.

Some specialized numerical schemes were developed to capture the specific kinematics associated with the presence of cracks. For instance, integrated approaches [14] were introduced when using Williams' kinematic series [15]. Since the introduction of FE-based DIC [16, 17, 18] and following on the development of the extended finite element method [19], the same type of kinematic enrichment was considered in extended DIC (X-DIC) [20, 21]. It was later generalized to $3 \mathrm{D}$ analyses of cracks monitored via computed microtomography $[22,23]$. Another route is 'a priori' node splitting [24, 25, 26, 27]. In both approaches (i.e., X-DIC and node splitting), it was shown that gray level residuals were very useful to adapt the level set function or the mesh to the crack path [28, 21, 23, 24, 26, 27] since they are evaluated pixel-wise.

The design of the mesh is crucial for FE-based methods, e.g., numerical simulation or DIC, especially in the presence of localized phenomena. The first FE-based DIC algorithms were pyramidal to enable for better convergence of the minimization scheme [18, 28, 29]. Such h-refinement procedure has to be dealt with care since DIC analyses are inverse problems, namely, the finer the discretization, the higher the measurement uncertainty [18, 30], yet the interpolation error is lower [28]. Heterogeneous h-refinement is appealing when dealing with localized phenomena such as strained bands [31] or cracks [32,33] to better capture high strain gradients [34]. As before, one critical aspect is related to the criterion for selecting elements to be refined. Several choices are possible, among them strains [32, 33] and gray level residuals (GLR) [31, 32, 27].

Another route is given by p-refinement, which is generally easier to implement since it does not affect the mesh connectivity. This procedure has already been used in DIC [35, 36], increasing the resolution without reducing the element size. Such implementations can be self-adaptative [37, 38]. Kleinendorst et al. [39] implemented p-refined DIC using Non Uniform Rational Basis Splines (NURBS).

Hereafter, the numerical procedures are first presented, i.e., DIC for the measurements, the Lagrange multipliers in the DIC scheme to deal with the hanging nodes, and the proposed adaptive meshing procedure. Then, three cases are shown to illustrate the Adaptative Meshing (AM) procedure. For the artificial case (\#1.1 to \#1.3), three images were created to compare the gains of applying AM with different crack densities. Then two experiments are analyzed, 
namely, a Wedge Splitting Test (WST, Case \#2) dealing with a dominant crack, and curing and drying of an MgO refractory castable (Case \#3) leading to the formation of a crack network, where the effects of AM over the DIC results are highlighted. For all cases, the locally refined meshes are compared with their analog uniform mesh (UM) with the smallest element size.

\section{Numerical procedures}

\subsection{Digital Image Correlation}

DIC is a full-field measurement technique based on the optical flow, i.e., the gray level $f$ of any pixel location $\mathbf{x}$ in the reference configuration is equal to the gray level $g$ in the deformed state image $g$ at location $\mathbf{x}+\mathbf{u}(\mathbf{x})$ instead of $\mathbf{x}$. The displacement field $\mathbf{u}$ is determined by minimizing the gray level residual $\Phi_{c}^{2}$ over the selected domain $\Omega$

$$
\Phi_{c}^{2}=\sum_{\Omega}[f(\mathbf{x})-g(\mathbf{x}+\mathbf{u}(\mathbf{x}))]^{2}
$$

Searching for $\mathbf{u}$ pixel-wise is an ill-posed problem, and thus it is regularized by searching in a subspace made of trial fields $\Psi_{k}$

$$
\mathbf{u}(\mathbf{x})=\sum_{p} a_{p} \Psi_{\mathbf{p}}(\mathbf{x})
$$

where $a_{p}$ are nodal displacements gathered in the column vector $\{\mathbf{a}\}$. The minimization of $\Phi_{c}^{2}(\{\mathbf{a}\})$ is carried out with an iterative Gauss-Newton scheme that leads to linear systems

$$
[\mathbf{H}]\{\delta \mathbf{a}\}=\{\mathbf{h}\}
$$

where $\{\delta \mathbf{a}\}$ gathers the updates to the nodal displacement for the current iteration, and the other terms are the Hessian matrix

$$
H_{i j}=\sum_{\Omega}\left(\Psi_{i} \cdot \nabla f\right)(\mathbf{x})\left(\Psi_{j} \cdot \nabla f\right)(\mathbf{x})
$$

and the residual vector

$$
h_{i}=\sum_{\Omega}\left[f(\mathbf{x})-g\left(\mathbf{x}+\widetilde{a}_{p} \Psi_{\mathbf{p}}(\mathbf{x})\right)\right]\left(\Psi_{i} \cdot \nabla f\right)(\mathbf{x})
$$

where $\tilde{a}_{p}$ is the current estimate of the nodal displacement component $a_{p}$.

Depending on the choice of domain $\Omega$ different correlation procedures are obtained. When $\Omega$ reduces to a small interrogation window, the previous setting corresponds to that of local DIC $[5,3]$. Conversely, $\Omega$ may cover the whole region of interest (ROI). In that case case, global approaches are obtained [40]. Among many choices for kinematic bases, finite element descriptions are very versatile $[17,18]$. In the present case, three noded (T3) triangles were considered (i.e., T3-DIC) [41]). 
In the present studies, very fine meshes will be constructed. At one stage, the discretization will be too fine for standard FE-based DIC to converge. It is however possible to supplement DIC with mechanical input, namely, via mechanical regularization [42, 43] based upon the equilibrium gap method [44]. In linear elasticity, the discretized equilibrium equations read

$$
[\mathbf{K}]\{\mathbf{a}\}=\{\mathbf{f}\},
$$

where $[\mathbf{K}]$ is the stiffness matrix, and $\{\mathbf{f}\}$ the vector of nodal forces. If the displacement field $\mathbf{u}$ does not satisfy equilibrium, force residuals $\left\{\mathbf{f}_{\mathbf{r}}\right\}$ arise

$$
\left\{\mathbf{f}_{\mathbf{r}}\right\}=[\mathbf{K}]\{\mathbf{a}\}-\{\mathbf{f}\}
$$

In the absence of body forces, the nodal forces of inner nodes must vanish, which makes the goal of the equilibrium gap approach to minimize [44]

$$
\Phi_{m}^{2}=\left\|\left\{\mathbf{f}_{\mathbf{r}}\right\}\right\|^{2}=\{\mathbf{a}\}^{\top}[\mathbf{K}]^{\top}[\mathbf{K}]\{\mathbf{a}\}
$$

where ${ }^{\top}$ is the transpose operator, and $\Phi_{m}^{2}$ the summation of the L2-norm of all equilibrium gaps for inner nodes and traction-free boundaries (i.e., Neumann nodes). For Dirichlet boundary nodes, the discrete Laplace-Beltrami operator is considered instead [45]. In the present case, the previous regularization was applied to all nodes.

To simultaneously reduce the correlation residuals $\Phi_{c}^{2}$ and the equilibrium gap $\Phi_{m}^{2}$, the total cost function $\Phi_{t}^{2}$

$$
\left(1+\omega_{m}\right) \Phi_{t}^{2}=\widehat{\Phi}_{c}^{2}+\omega_{m} \widehat{\Phi}_{m}^{2}
$$

is minimized, where $\omega_{m}$ is the weight that defines the scale associated with the equilibrium gap, $\widehat{\Phi}_{c}^{2}$ and $\widehat{\Phi}_{m}^{2}$ normalized cost functions. The importance of normalization is to convert the residuals into dimensionless quantities [46, 45]. It is carried out by considering a trial displacement field in the form of a plane wave $\mathbf{v}(\mathbf{x})=\mathbf{v}_{\mathbf{0}} \exp (i \mathbf{k} \cdot \mathbf{x})$, where $\mathbf{v}_{\mathbf{0}}$ is the amplitude and $\mathbf{k}$ the wave vector. The normalized cost functions become

$$
\widehat{\Phi}_{c}^{2}=\frac{\Phi_{c}^{2}}{\{\mathbf{v}\}^{\top}[\mathbf{H}]\{\mathbf{v}\}} \quad, \quad \widehat{\Phi}_{m}^{2}=\frac{\phi_{m}^{2}}{\{\mathbf{v}\}^{\top}[\mathbf{K}]^{\top}[\mathbf{K}]\{\mathbf{v}\}} .
$$

The wavelength dependence of $\{\mathbf{v}\}^{\top}[\mathbf{K}]^{\top}[\mathbf{K}]\{\mathbf{v}\}$ is of the fourth-order wrt. $\|\mathbf{k}\|$, and the quantity $\{\mathbf{v}\}^{\top}[\mathbf{H}]\{\mathbf{v}\}$ is independent of the wave number $\|\mathbf{k}\|$. The weight reads

$$
\omega_{m}=\left(\|\mathbf{k}\| \ell_{m}\right)^{4}
$$

where $\ell_{m}$ is the so-called regularization length (for $\Phi_{m}^{2}$ ). Therefore, the weight put on the cost function is proportional to $\ell_{m}$ raised to the power 4 . The value chosen for $\ell_{m}$ should be small enough to fit in small ROIs but large enough to avoid divergence of the minimization scheme [46]. For this work, whose objective is to monitor cracks, the value 
of $\ell_{m}$ needs to be as small as possible, otherwise displacement jumps in the crack path will be spread over adjacent elements.

Instead of solving Systems (3), the new systems become

$$
\left[\mathbf{H}^{t}\right]\{\delta \mathbf{a}\}=\left\{\mathbf{h}^{t}\right\}
$$

with

$$
\left[\mathbf{H}^{t}\right]=[\mathbf{H}]+\omega_{m} \frac{\{\mathbf{v}\}^{\top}[\mathbf{H}]\{\mathbf{v}\}}{\{\mathbf{v}\}^{\top}[\mathbf{K}]^{\top}[\mathbf{K}]\{\mathbf{v}\}}[\mathbf{K}]^{\top}[\mathbf{K}]
$$

and

$$
\left\{\mathbf{h}^{t}\right\}=\{\mathbf{h}\}-\omega_{m} \frac{\{\mathbf{v}\}^{\top}[\mathbf{H}]\{\mathbf{v}\}}{\{\mathbf{v}\}^{\top}[\mathbf{K}]^{\top}[\mathbf{K}]\{\mathbf{v}\}}[\mathbf{K}]^{\top}[\mathbf{K}]\{\tilde{\mathbf{a}}\}
$$

The iterations are stopped when the L2-norm of the vector containing the root mean square (RMS) of the horizontal and vertical displacement corrections was less than $10^{-3}$ px.

\subsection{Adaptive meshing}

\subsubsection{Refinement strategy}

In the present work, the mesh size will be lowered within FE-based DIC procedures (i.e., heterogeneous h-refinement). 73 During a local h-refinement, some nodes located in the middle of an edge between two elements (e.g., one subdivided $\mathbf{7 4}$ and others not), have different numbers of connectivities. To illustrate this phenomenon, let us consider a structured $\mathbf{7 5}$ mesh with two triangular elements (Figure 1(a)). If each element is divided into four new elements of the same size, 76 there is no lack of connectivity at any node, as shown in Figure 1(b). However, if only the element 1-4-3 is subdivided into four new ones, it will generate one hanging node (i.e., node 6 in Figure 1(c) is a hanging node in element 1-2-4), 78 which does not have the correct number of connectivities (see node 6 in Figure 1(b)). Nodes 5 and 7 in Figure 1(c) 79 are not hanging nodes because they are on the outer edges. For localized h-refinement, at least one node is created on the edge that separates the subdivided domain and the intact one. The presence of hanging nodes makes the mesh non-conformal, which can induce numerical artifacts. 


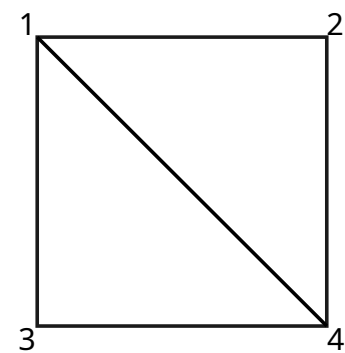

(a)

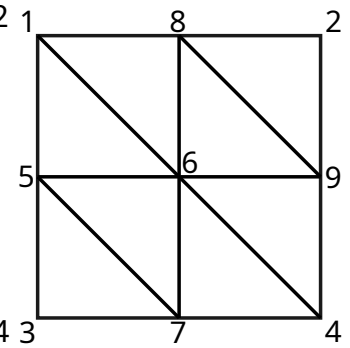

(b)

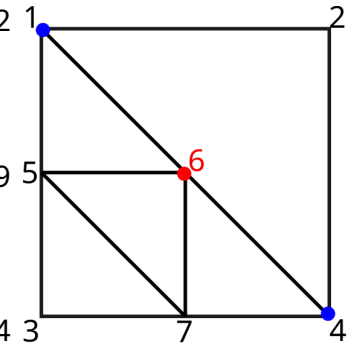

(c)

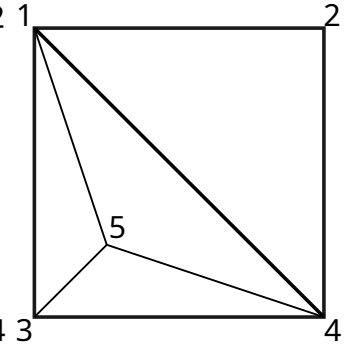

(d)

Figure 1: Examples of various localized h-refinements. (a) Original mesh. (b) Global h-refinement. (c) Local refinement (non conformal mesh). (d) Local refinement with conformal mesh

A natural alternative to deal with hanging nodes is to divide the elements creating a new node at the centroid of a parent element and connect it to the element vertices (Figure 1(d)). However, this approach generates slender elements that may compromise the calculations. To overcome this drawback, Baldi and Bertolino [32] reconstructed the mesh using the new nodes provided by local refinement. An alternative solution to remeshing strained regions (in the present case cracks) is proposed herein, which uses Lagrange multipliers to link the displacement of hanging nodes based on those of the adjacent (also called parent) nodes (nodes 1 and 4 in Figure 1) and the shape function of the considered element.

If an element edge is divided at its middle point, the displacements of the hanging node is the average of the displacement of its parent nodes. If the edges are not split in their middle, weights related to the distance from the hanging node to each parent node should be considered and the shape functions. Such an approach can be easily implemented in a DIC algorithm and does not require the DIC Hessian to be changed, because only an auxiliary (Lagrange) matrix $[\mathbf{L}]$ is added. The $[\mathbf{L}]$ matrix is pre-multiplied by the ratio of Frobenius norms of $\left[\mathbf{H}^{t}\right]$ and $[\mathbf{L}]$, $\alpha$, to avoid degrading the full system conditioning. Once all the desired constraints are accounted for, the augmented Hessian $\left[\mathbf{H}^{a}\right]$ becomes

$$
\left[\mathbf{H}^{a}\right]=\left[\begin{array}{c|c}
{\left[\mathbf{H}^{t}\right]} & \alpha[\mathbf{L}]^{\top} \\
\hline \alpha[\mathbf{L}] & {[\mathbf{0}]}
\end{array}\right]
$$

The vector of unknowns $\{\boldsymbol{\beta}\}$ now gathers all nodal displacements $\{\mathbf{a}\}$ and Lagrange multipliers $\{\boldsymbol{\lambda}\}$, and the augmented residual vector $\left\{\mathbf{h}^{a}\right\}$ reads

$$
\left\{\mathbf{h}^{a}\right\}^{\top}=\left\{\left\{\mathbf{h}^{t}\right\}^{\top}\{\mathbf{0}\}^{\top}\right\}
$$

resulting in the augmented system to solve as

$$
\left[\mathbf{H}^{a}\right]\{\delta \boldsymbol{\beta}\}=\left\{\mathbf{h}^{a}\right\}
$$


In experimental cases, defining which elements need to be divided is not straightforward. There are several choices, among them the maximum eigen strains [34, 32, 33] and the gray level residuals [28, 21, 24, 26, 31, 34, 27]. It is worth noting that the eigen strains are computed as the average over each element. Conversely, the gray level residuals $\rho(\mathbf{x})=f(\mathbf{x})-g\left(\mathbf{x}+v_{p}^{m} \Psi_{\mathbf{p}}(\mathbf{x})\right)$, where $v_{p}^{m}$ are the measured nodal displacements (i.e., at convergence of the DIC code), are defined at the pixel scale. In both cases, a user-defined threshold will be needed.

\subsubsection{Refinement Criterion}

Since cracks are to be detected and quantified, it is proposed to introduce a refinement criterion explicitly accounting for displacement jumps and their associated measurement uncertainty. In the presence of cracks, the mean strain tensor in each element is expressed as [47]

$$
\overline{\boldsymbol{\epsilon}}=\frac{1}{\left|\Omega_{e}\right|} \int_{\Omega_{e}} \boldsymbol{\epsilon}(\mathbf{x}) \mathrm{d} \mathbf{x}+\frac{1}{\left|\Omega_{e}\right|} \int_{\Gamma_{e}} \llbracket \mathbf{u}(\mathbf{x}) \rrbracket \bigotimes \mathbf{n}(\mathbf{x}) \mathrm{d} \mathbf{s}
$$

where $\llbracket \mathbf{u} \rrbracket$ denotes the displacement jump, $\mathbf{n}$ the normal to the cracked surface $\Gamma_{e}, \nabla$ the symmetrized tensorial product, $\boldsymbol{\epsilon}$ the strain tensor of the uncracked matrix, and $s$ the curvilinear abscissa along the crack path. In the following, it $\mathbf{1 0 0}$ is assumed that the elastic strain levels are negligible with respect to the singular contribution provided by cracks. 101 Consequently, the mean strain tensor reduces to

$$
\overline{\boldsymbol{\epsilon}} \approx \frac{1}{\left|\Omega_{e}\right|} \int_{\Gamma_{e}} \llbracket \mathbf{u}(\mathbf{x}) \rrbracket \bigotimes \mathbf{n}(\mathbf{x}) \mathrm{d} \mathbf{s}
$$

The mean crack opening displacement $\overline{\llbracket \mathbf{u} \rrbracket}$ per element is defined as

$$
\overline{\llbracket \mathbf{u} \rrbracket} \otimes \overline{\mathbf{n}}=\frac{1}{\left|\Gamma_{e}\right|} \int_{\Gamma_{e}} \llbracket \mathbf{u} \rrbracket \otimes \mathbf{n} \mathrm{d} \mathbf{s}
$$

where $\overline{\mathbf{n}}$ denotes the mean crack normal. Further, it is assumed that the main opening contribution is associated with the mode I regime

$$
\overline{\llbracket \mathbf{u} \rrbracket}=\overline{\llbracket u \rrbracket} \overline{\mathbf{n}}
$$

such that $\overline{\mathbf{n}}$ then corresponds to the eigen direction associated with the average maximum eigen strain $\epsilon_{1}$. The latter is then related to the mean crack opening displacement $\overline{\llbracket u \rrbracket}$ by

$$
\left|\Omega_{e}\right| \epsilon_{1}=\left|\Gamma_{e}\right| \overline{\llbracket u \rrbracket}
$$

It is worth noting that $\left|\Omega_{e}\right| /\left|\Gamma_{e}\right|$ scales with the element size $L$, provided only one crack is present in each element. 108 This hypothesis is likely to be true for small element sizes. Consequently, the mean crack opening displacement is approximated by

$$
\overline{\llbracket u \rrbracket}=L \epsilon_{1}
$$


The mean crack opening displacement threshold $\llbracket u^{*} \rrbracket$ will be set to classify elements as damaged (i.e., $\overline{\llbracket u \rrbracket} \geq \llbracket u^{*} \rrbracket$ ). It is proposed to relate $\llbracket u^{*} \rrbracket$ to its measurement uncertainty (i.e., typically 5 times or more its standard uncertainty $\sigma\left(\overline{\llbracket u \rrbracket)}\right.$ ). According to Equation (23), the uncertainty $\sigma(\overline{\llbracket u \rrbracket})$ is related to the standard strain uncertainty $\sigma\left(\epsilon_{1}\right)$ by $\sigma(\overline{\llbracket u \rrbracket})=L \sigma\left(\epsilon_{1}\right)$. Further, the strain uncertainty $\sigma\left(\epsilon_{1}\right)$ is dependent on the element size

$$
\sigma\left(\epsilon_{1}\right)=A L^{\alpha}
$$

and characterizes the compromise to be carried out between the uncertainty and the discretization level (i.e., spatial resolution). Typical values of the power $\alpha$ range between -1 and -2 (i.e., when the measurement uncertainties are random and controlled by white Gaussian noise [48]). The constant $A$ is dependent on the contrast in the picture and the noise level [18]. The standard uncertainty $\sigma\left(\epsilon_{1}\right)$ (and therefore $\sigma(\overline{\llbracket u \rrbracket)})$ is obtained by performing an uncertainty quantification prior to each analyzed test. Consequently, the threshold $\llbracket u^{*} \rrbracket$ becomes

$$
\llbracket u^{*} \rrbracket(k)=k A L^{\alpha+1}
$$

where $k$ is a proportionality constant between the standard uncertainties. Thus, the threshold $\llbracket u^{*} \rrbracket$ explicitly depends

By using Equation (23), a strain threshold $\epsilon^{*}(L)=\llbracket u^{*} \rrbracket(L) / L$ can also be defined. With the previous proposition, 116 it is concluded that $\epsilon^{*}$ is also length dependent. This proposition departs from the use of a unique strain threshold [31, 117 $32,33]$ when multiple refinement steps are to be performed. Both thresholds (i.e., $\epsilon_{1}^{*}$ and $\left.\llbracket u^{*} \rrbracket\right)$ introduced herein relate to the measurement uncertainties of the associated quantities (i.e., $\epsilon_{1}$ and $\overline{\llbracket u \rrbracket}$ ). Throughout this work the discussion will be focused on the use of $\llbracket u^{*} \rrbracket$ and its dependence on the parameter $k$.

\subsubsection{Refinement Steps}

It is proposed to start off with a coarse mesh and to use the previous criterion to refine the 'damaged' elements

1. perform a DIC analysis using a coarse uniform mesh (UM);

2. choose an image for crack evaluation based on the displacement jump $(\llbracket u \rrbracket)$;

3. list the damaged elements for this image pair and considered discretization;

4. execute one subdivision in the damaged elements (i.e., each selected element generates four new ones);

5. identify the hanging nodes and respective parent nodes; 
6. construct the Lagrange matrix and perform DIC analyses for this image pair using the locally refined mesh;

7. repeat items 3 to 6 until the smallest elements achieve the size desired by the user;

8. perform a DIC analysis for all images using the optimized mesh.

This approach was implemented within the Correli 3.0 framework [49] and is described in the flowchart of Figure 2. 133 It is worth noting that, in the present implementation, the end of the adaptive procedure is a user decision, utilizing the size of the smallest element after division as a stop criterion. The smallest element size may be guided by the highest measured Mean Crack Opening Displacement (MCOD) using UMs. If even smaller elements were envisaged (i.e., down to one pixel size), damage could be used along with mechanical regularization [34].

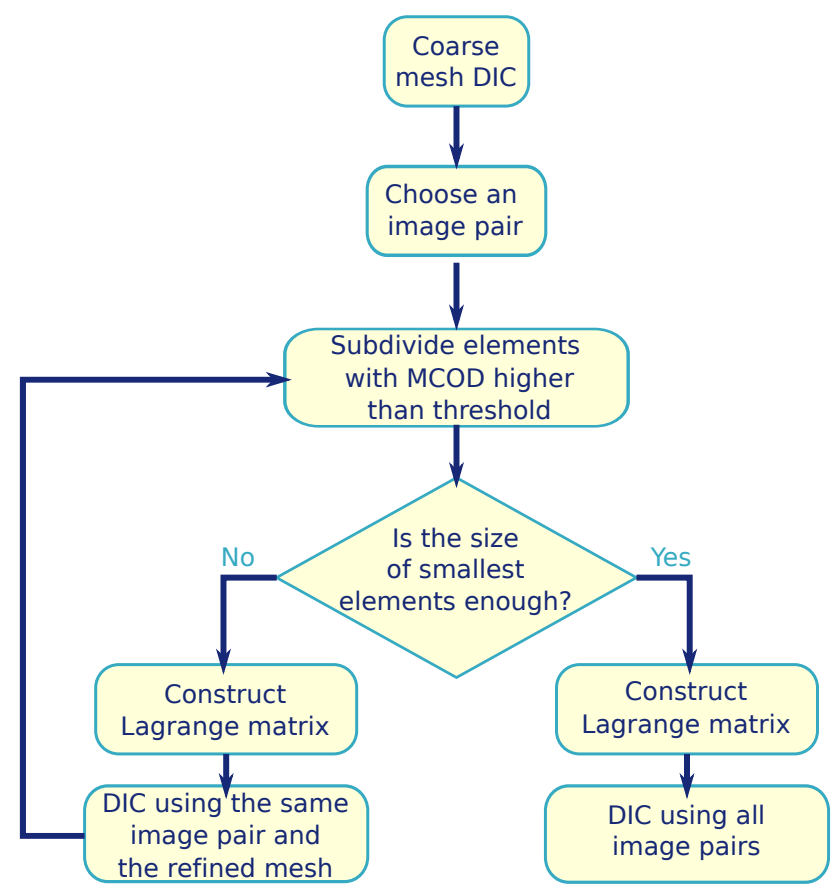

Figure 2: Flowchart of the AM procedure applied to DIC, using MCOD as division criterion

\section{Artificial Cases (\#1.1 to \#1.3)}

This statement is specific to the artificial images .

The artificial images of $200 \times 200$ pixel definition shown in Figure 3 are analyzed to illustrate the benefits of the adaptive meshing proposal. Such pictures may correspond to the gray level residuals at the end of a DIC run [28, $21,23,24,26,27]$. In the present cases these images were built to demonstrate the method (i.e., the threshold for binarization is known), the binary images remove the user-dependence to identify the cracked elements. To represent practical situations, a single crack (Case \#1.1, Figure 3(a)) is assumed with a sinuous front. An additional case consists of a major macrocrack with some branches (Case \#1.2, Figure 3(b)). Last, a crack network is considered (Case \#1.3, 145 
Figure 3(c)). The corresponding damaged areas (i.e., ratio of cracked pixels wrt. total number of pixels) is equal to 1, 146 3 , and $15 \%$, respectively.

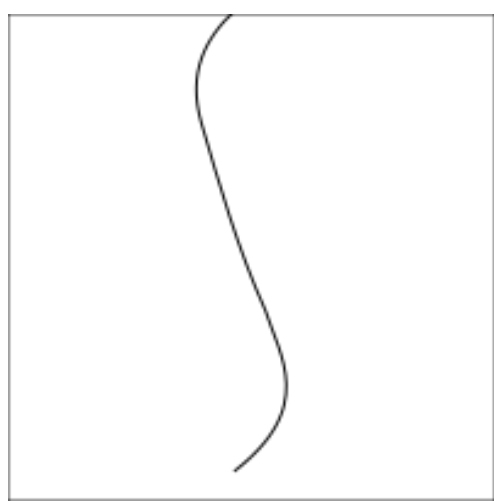

(a)

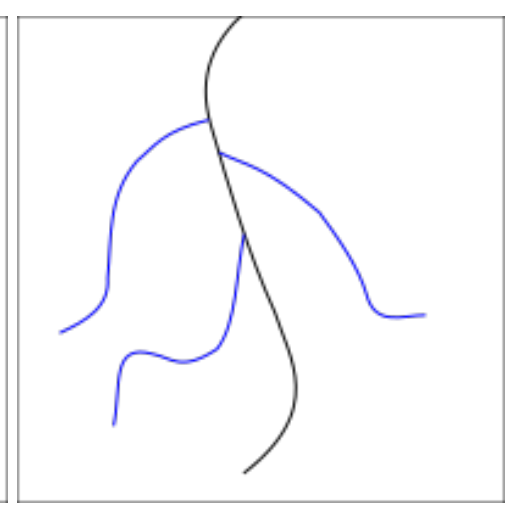

(b)

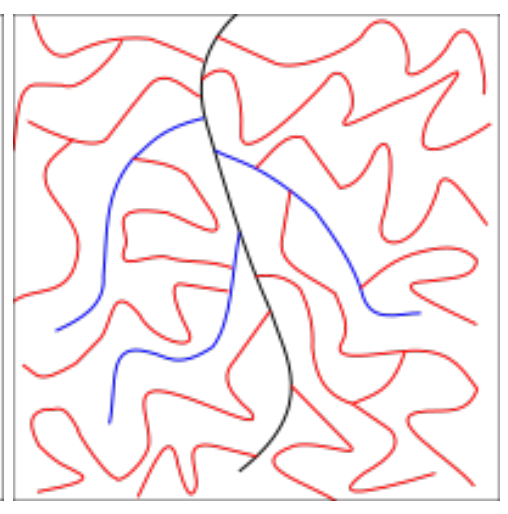

(c)

Figure 3: Artificial images analyzed herein. (a) Single crack (Case \#1.1). (b) Major crack with branchings (Case \#1.2).

(c) Crack network (Case \#1.3)

These images are first binarized, in which one represents a cracked state, and zero otherwise. Then, starting from 148 the simple mesh shown in Figure 4(a), if any pixel that represents a crack is contained in an element, it is divided into four smaller elements (see Figure 1(c)), and this procedure is iteratively repeated. The starting element is of the image 150 size, with a mean element size $L_{0}=141 \mathrm{px}$, obtained as the square root of the element area $\left(\sqrt{A_{0}}\right)$. Hereafter, the nomenclature $L_{\mathrm{it}}$ will be used for the mean element size of iteration it for the reference case where all elements are divided, with it $=0$ the initialization. It is worth noting that $L_{\mathrm{it}}$ is also related to the smallest element size in the AM 153 methodology proposed herein. Seven iterations were chosen for the smallest element to remain greater than 1 px (i.e., $L_{7}=1.1 \mathrm{px}$ in the present case). Figure 4 shows the starting mesh along with the first two iterations of the reference mesh, i.e., the case where all elements were subdivided. 


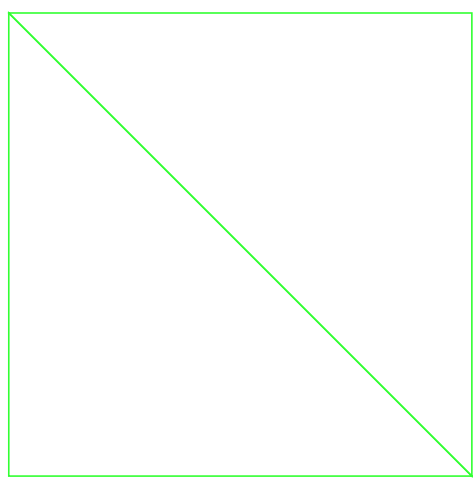

(a)

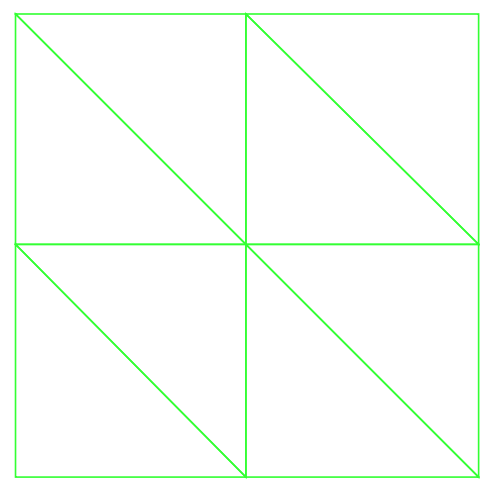

(b)

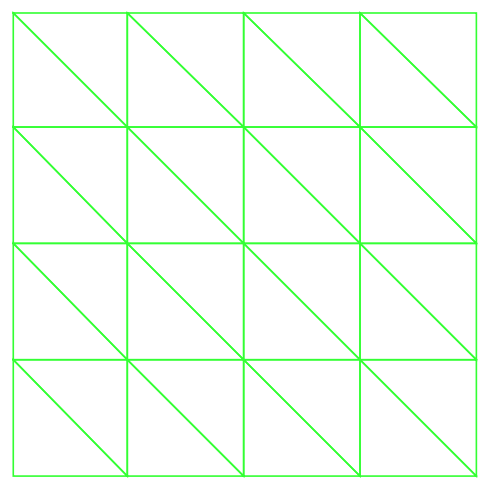

(c)

Figure 4: Reference and first uniform meshes used in the artificial cases. (a) Starting mesh ( $\left.L_{0}=141 \mathrm{px}\right)$. (b) First iteration $\left(L_{1}=70 \mathrm{px}\right)$. (c) Second iteration $\left(L_{2}=35 \mathrm{px}\right)$

After seven refinements, the resulting meshes for the three cases are shown in Figure 5. It is possible to see how some elements remained undivided in undamaged regions, especially for the first and second cases (Figure 5(a-b)), while very small elements were obtained in the cracked regions $\left(L_{7} \approx 1.1 \mathrm{px}\right)$.

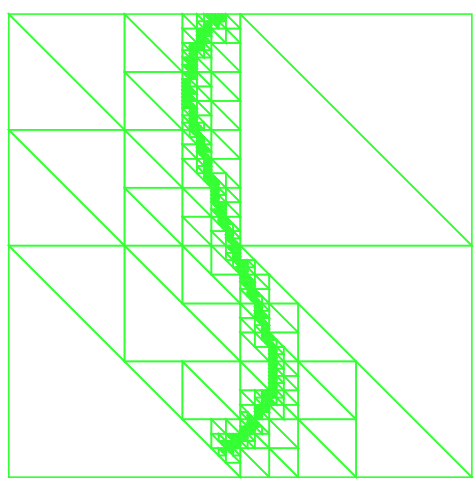

(a)

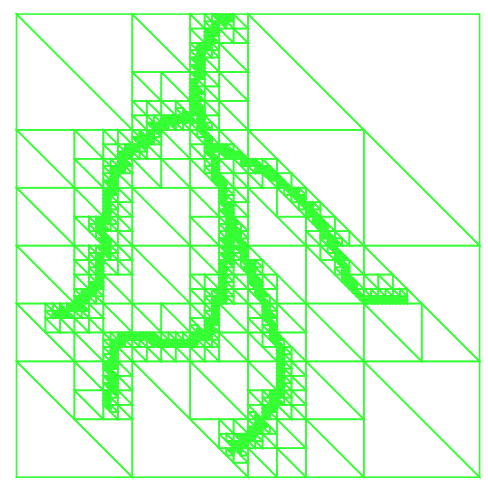

(b)

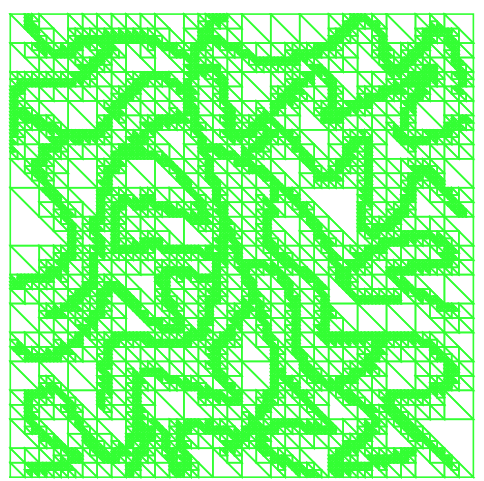

(c)

Figure 5: Meshes for the three artificial cases after 7 refinements (Figure 3). (a) Case \#1.1 (b) Case \#1.2 (c) Case \#1.3

In the case of the reference meshes where all elements were subdivided, the DIC Hessian matrix [H] was also 160 computed for comparison purposes. For three refined meshes, the augmented Hessians $\left[\mathbf{H}^{a}\right]$ were assembled to also 161 consider the new constraints using Lagrange multipliers. The sizes of the Hessian matrices are shown in Table 1 for all 162 three cases. In virtually all cases, there is a clear benefit of the AM procedure, especially for very fine discretizations. 


\section{Table 1}

Size of Hessian matrices as a function of smallest element sizes $L_{\mathrm{it}}$ for the artificial cases

\begin{tabular}{|c|c|c|c|c|c|}
\hline iteration & $L_{\text {it }}[\mathrm{px}]$ & reference & Case \#1.1 & Case \#1.2 & Case \#1.3 \\
\hline \hline 1 & 70 & 27 & 27 & 27 & 27 \\
\hline 2 & 35 & 75 & 63 & 72 & 75 \\
\hline 3 & 18 & 243 & 147 & 204 & 243 \\
\hline 4 & 8.8 & 867 & 303 & 558 & 882 \\
\hline 5 & 4.4 & 3,267 & 639 & 1,416 & 3,552 \\
\hline 6 & 2.2 & 12,675 & 1,335 & 3,219 & 11,661 \\
\hline 7 & 1.1 & 49,923 & 2,856 & 7,278 & 31,008 \\
\hline
\end{tabular}

The relative gains in terms of size of the sparse matrices are reported in Table 2 when compared to uniformly refined $\mathbf{1 6 4}$ solution, calculated as one minus each Hessian size divided by its reference for a given iteration. There were negative 165 values in some iterations, only for the crack network (i.e., because more hanging nodes were created than edges that 166 had not to be split). The gains of this refinement methodology are inversely proportional to the crack density. In cases $\mathbf{1 6 7}$ with few cracks, it is interesting to have fewer elements where no localized phenomena are taking place, for a more 168 efficient calculation and no loss of accuracy. However, for very dense crack networks, this approach may even be worse $\mathbf{1 6 9}$ in some cases than a full mesh refinement (e.g., when $L \approx 10 \mathrm{px}$ ). If very small elements are required (i.e., about the 170 pixel size), it is likely that this method allows for gains in most cases.

\section{Table 2}

Percent gains on size of the Hessian matrices in comparison to the reference case.

\begin{tabular}{|c|c|c|c|c|}
\hline iteration & $L_{\mathrm{it}}[\mathrm{px}]$ & Case \#1.1 & Case \#1.2 & Case \#1.3 \\
\hline \hline 1 & 70 & 0 & 0 & 0 \\
\hline 2 & 35 & 16 & 4 & 0 \\
\hline 3 & 18 & 40 & 16 & 0 \\
\hline 4 & 8.8 & 65 & 36 & -2 \\
\hline 5 & 4.4 & 80 & 57 & -9 \\
\hline 6 & 2.2 & 89 & 75 & 8 \\
\hline 7 & 1.1 & 94 & 85 & 38 \\
\hline
\end{tabular}




\section{Experimental Cases}

\subsection{Wedge Splitting Test (Case \#2)}

One experimental setup that allows for stable crack propagation is the so-called Wedge Splitting Test (WST) [50, 174

51]. It is used hereafter to exemplify the benefits of the method proposed herein when not too many cracks are suspected $\mathbf{1 7 5}$ to occur. In the WST, a vertical force is transmitted to a pre-notched sample using a wedge and cylinders to prescribe $\mathbf{1 7 6}$ a horizontal (mode I) splitting load. DIC was used to analyze WST experiments via subset-based DIC [52, 53, 54, 55] 177 or using T3-DIC [56, 57] based on structured meshes. Further, Ref. [58] discussed how to calibrate a cohesive zone 178 model for such experiment, which required a mesh to be adapted in the crack propagation zone.

The studied material was a commercial castable refractory DD40, made by IBAR, that can be utilized in catalytic 180 cracking units of oil industries [59, 60, 61]. Wedge Splitting Tests (WSTs), which were performed along the guidelines proposed in Ref. [62], were first analyzed via integrated DIC [56]. The specimen used in the test reported hereafter 182 was similar with the sole difference being the absence of lateral grooves to prescribe straight propagation. A parallelepipedic sample of size $100 \times 100 \times 75 \mathrm{~mm}^{3}$ was molded with a pre-notch to insert the loading parts. The reference image and the initial mesh (in yellow) used in the procedure developed herein are shown in Figure 6(a). In the same 185 figure, the wedge, both cylinders, and blocks that were used to transmit the load horizontally, are also highlighted in 186 white dashed lines. A random pattern was created by spraying black and white paints to increase the image contrast. In the present experiment, there was no groove to guide the crack so that a tortuous crack path may be observed with 188 possibly some bifurcations. 


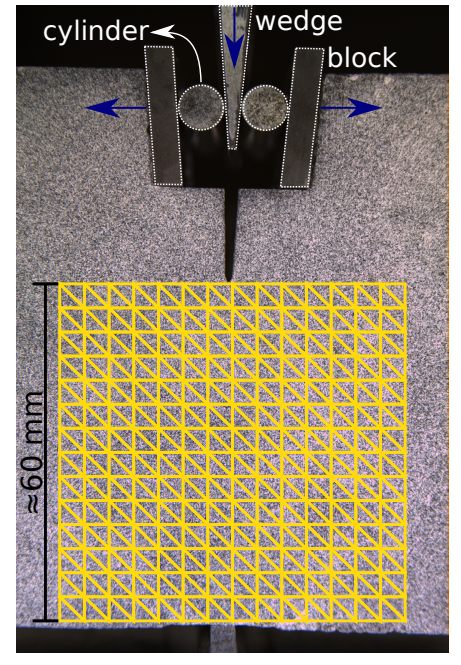

(a)

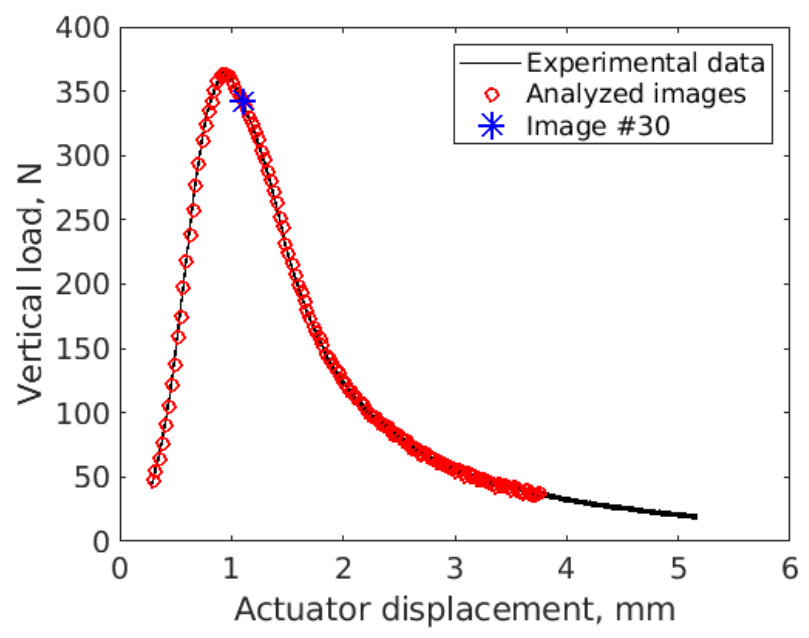

(b)

Figure 6: (a) Patterned specimen and coarse mesh in yellow (mean element size: $\approx 64 \mathrm{px}$ or $3 \mathrm{~mm}$ ) used for preliminary analyses. The loading parts are depicted in white dashed lines. The applied vertical force $F_{V}$ and the horizontally transmitted forces $\left(F_{H} \approx 5.7 F_{V}\right)$ to the sample are shown as blue arrows. (b) Load displacement curve for the studied WST down to $10 \%$ of the peak load. The red circles depict image acquisitions and the blue asterisk depicts the frame used in the discussion.

A pre-load of $40 \mathrm{~N}$ was applied before starting the test, to adjust the loading parts (Figure 6(b)). The experiment 190 was controlled with a constant actuator velocity of $80 \mu \mathrm{m} / \mathrm{min}$ and was stopped at $5 \%$ of the maximum load. A $0.1 \mathrm{~Hz} 191$ image acquisition rate was used (i.e., a total of 354 pictures was available). Only the odd images ranging from 5 to 257192 (i.e., down to $10 \%$ of the peak load) were analyzed to avoid lengthy discussions on the last part of the WST, resulting in a total of 126 images. The 30th analyzed frame (blue asterisk in Figure 6(b)) will be used throughout the discussion. 194 The DIC parameters used hereafter are reported in Table 3.

Table 3

DIC analysis parameters

\begin{tabular}{|l|l|}
\hline \hline DIC software & Correli 3.0 [49] \\
Image filtering & none \\
Shape functions & linear (T3) \\
Matching criterion & Regularized sum of squared differences \\
Interpolant & cubic \\
Strain calculation & derivative of shape functions \\
Regularization length & $20 \mathrm{px}$ \\
\hline
\end{tabular}


The five images acquired during the pre-loading step were used to compute the measurement uncertainties for

UMs. The temporal variances of the maximum eigen strain were computed in each element, and their root mean is shown in Figure 7(a) as a function of the inverse element size $L^{-1}$. The expected power law dependence described 198 by Equation (24) is observed with $\alpha=-1.66$. This value is close but not equal to -2 (i.e., some other sources of 199 uncertainties occurred in this experimental configuration). The standard MCOD uncertainty is reported in Figure 7(b). 200 A similar agreement is observed in comparison to Equation (25). The black stars in Figure 7 refer to the uncertainties computed for the optimal $\operatorname{AM}(k=3)$, and the procedure to achieve this adapted mesh is described hereafter.

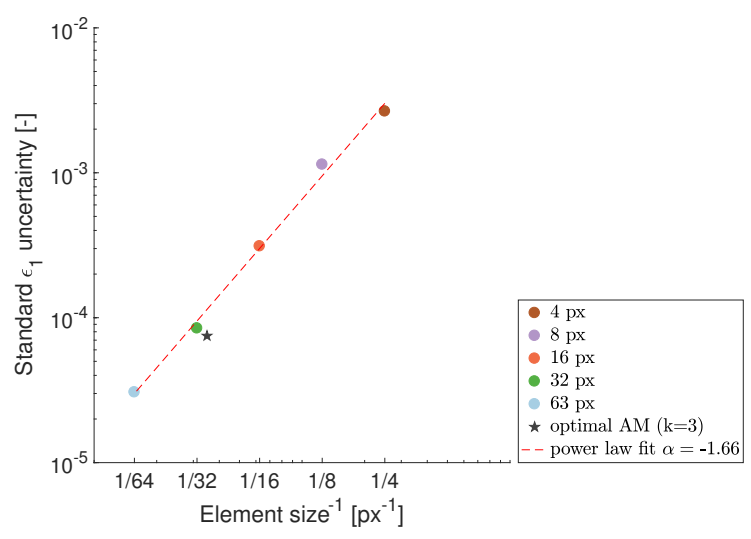

(a)

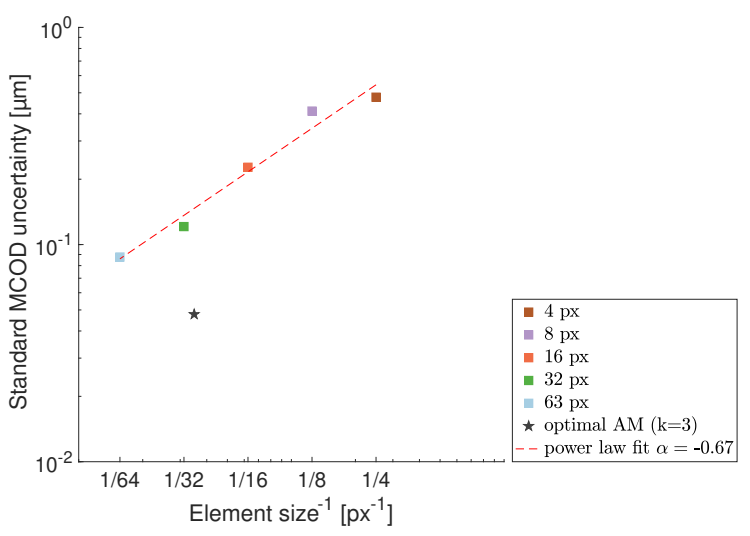

(b)

Figure 7: Standard uncertainties of the maximum eigen strain (a) and MCOD (b) as functions of the inverse element size $L^{-1}$ (UMs) for the initial images of the WST. The dashed lines depict power law fits according to Equations (24) and (25). The black stars mark the uncertainties for the optimal AM with $k=3$

For each frame, the standard deviation of the gray level residuals (GLR) defined as

$$
\rho_{c}(\mathbf{x})=f(\mathbf{x})-g(\mathbf{x}+\mathbf{u}(\mathbf{x}))
$$

was computed and normalized by the dynamic range of the reference picture (i.e., $\left.\Delta f=\max _{\mathrm{ROI}}(f)-\min _{\mathrm{ROI}}(f)\right), \mathbf{2 0 3}$ and the effect of the element size using UMs are shown in Figure 8. In the beginning of the test, the gray level 204 residuals were independent of the element size. Their overall level was related to acquisition noise, and the fluctuations due to small lighting variations. As the crack initiated and propagated, the residuals became more dependent on the element size (i.e., the larger the element size, the higher the residuals). This feature is explained by the lack of 207 degrees of freedom to properly describe the kinematics of the crack, which is improved by finer meshes. This effect $\mathbf{2 0 8}$ corresponds to interpolation errors [28]. Further, for small element sizes, the gain in discretization is less pronounced. 209 This phenomenon is due to the regularization scheme used herein, which dampens out sharp displacement gradients over the area defined by the regularization length. 


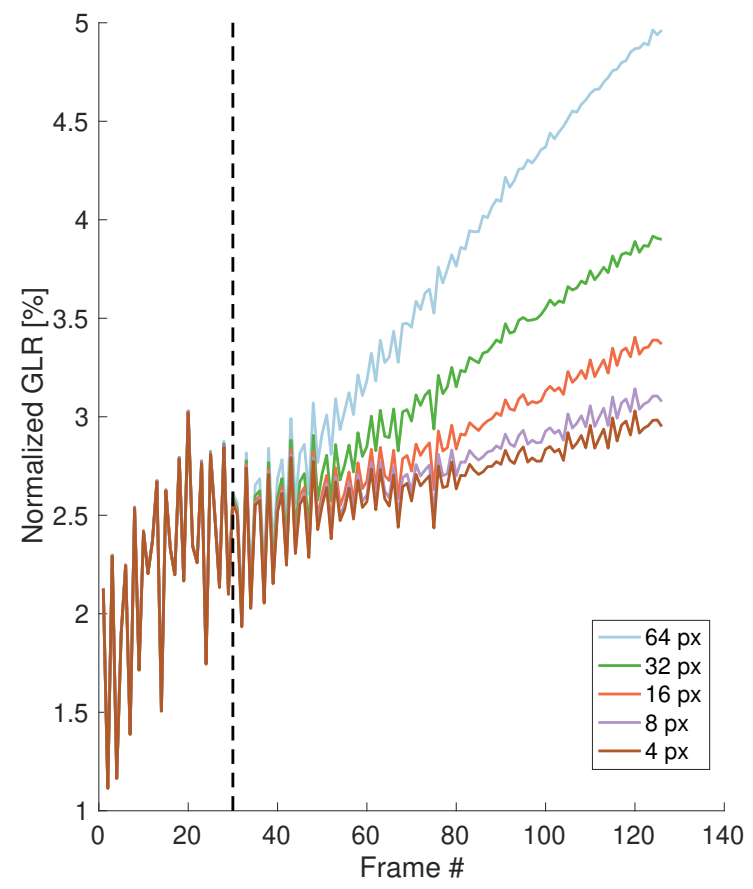

Figure 8: Normalized standard deviation of the gray level residuals (GLR) fields in each frame (\% of the dynamic range) for UMs with different element sizes. The vertical dashed line depicts frame \#30

GLR fields are shown in Figure 9 for the frame \#30. The GLR field of 64 px UM was set as a reference and the GLR 212 fields of the other UMs were subtracted from it to highlight their differences. As expected, the crack is better accounted 213 for as the element size decreases. However, using a very fine mesh implies not only higher computational cost, but 214 also more difficult convergence of the analysis due to the reduced number of pixels inside each element. This issue can 215 be addressed via regularization strategies (e.g., mechanical regularization [63, 64] as used in this work). Last, let us 216 note that even with very fine meshes, there are still high gray level residuals in the cracked region, which were induced 217 by the hypothesis of continuous displacement fields since no node splitting strategy was used herein [24, 25, 26, 27]. 218 One can notice gray level residuals almost spread over the entire ROI, which does not change with the element size. 219 The residuals are due to the acquisition noise and to fluctuations due to small lighting variations.

The mean crack opening displacement (MCOD) fields for frame \#30 computed using different element sizes are 221 shown in Figure 10. MCOD fields were less affected by the acquisition noise in comparison to the grainy GLR fields, 222 pointing MCOD as a better metric for crack detection. Further, crack details (e.g., bifurcations) are better captured by $\mathbf{2 2 3}$ small element sizes. However, it is essential to stress that uniform meshes with small element sizes require a higher $\mathbf{2 2 4}$ computational cost. This fact motivates the use of meshes whose refinement is "MCOD-guided" as discussed hereafter. 225 The refinement from 64-px UM down to 8-px UM showed a visible benefit to describe the crack and its ramification. 226 However, the 4-px UM does not present significant improvements when compared to 8 px UM (even with a four times 

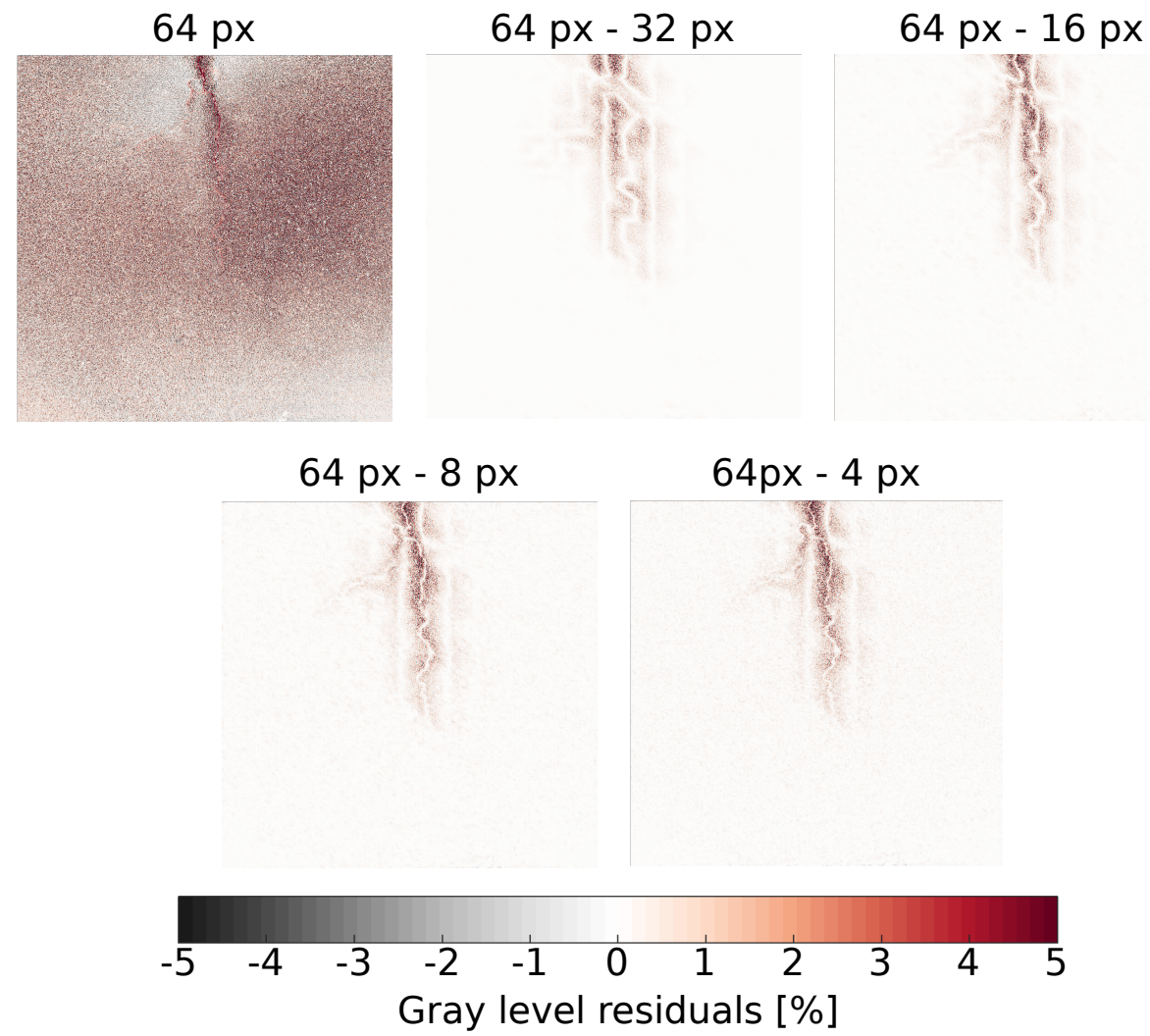

Figure 9: Normalized gray level residual field and differences for the frame \#30 of the analysis using UMs with different element sizes. For the sake of visualization the results from finer meshes were subtracted from the coarser mesh result (64 px). The color bar range was reduced to highlight the patterns in the field

larger Hessian, see Table 4). This observation is due to the regularization length utilized herein. Considering all the 228 previous points, the best compromise for the tested UMs is provided by the 8-px discretization. 


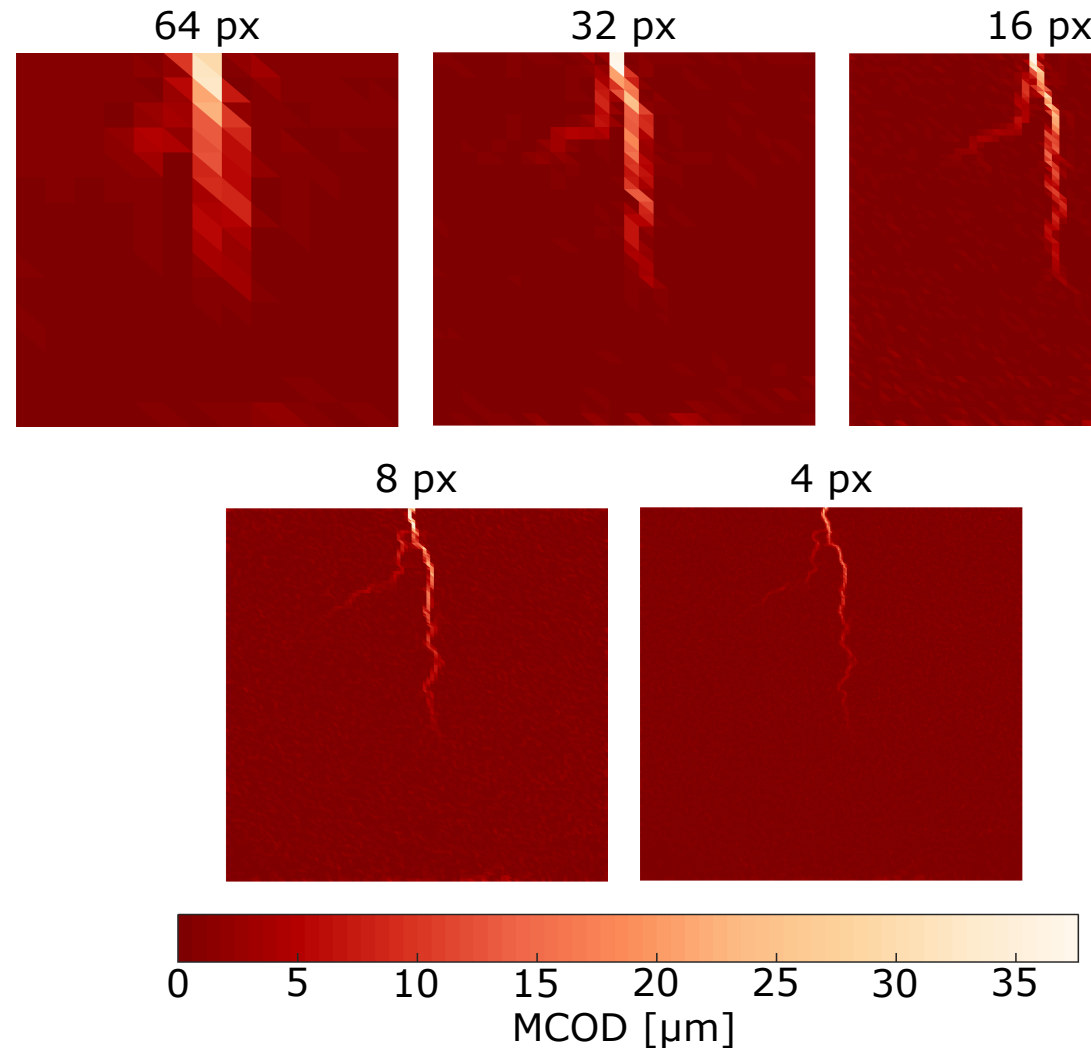

Figure 10: Mean crack opening displacement (MCOD) fields for the frame \#30 of the analysis using UMs with different element sizes $(1 \mathrm{px} \equiv 48.5 \mu \mathrm{m})$

The following analyses are devoted to adapted meshes. In Figure 11, the map of mesh sizes are reported at the end 230 of the adaption steps for 5 different levels of $k$ (i.e., the ratio between the measurement uncertainty and the threshold 231 used to detect damaged elements). When the threshold is very high (i.e., $k=12,6$ ), only the zones surrounding the 232 main crack are refined. Conversely, when $k$ is too low (e.g., $k=1.5)$, the mesh is refined in areas that are not cracked. 233 From these first analyses, it is concluded that $k=3$ leads to the best compromise. 


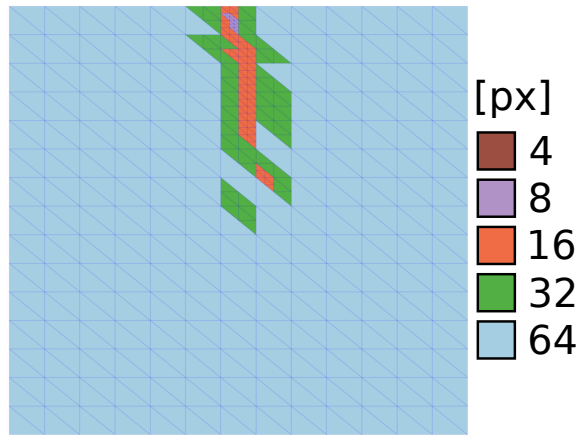

(a)

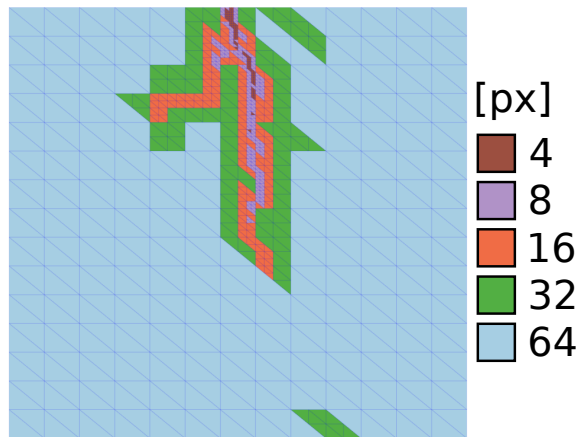

(c)

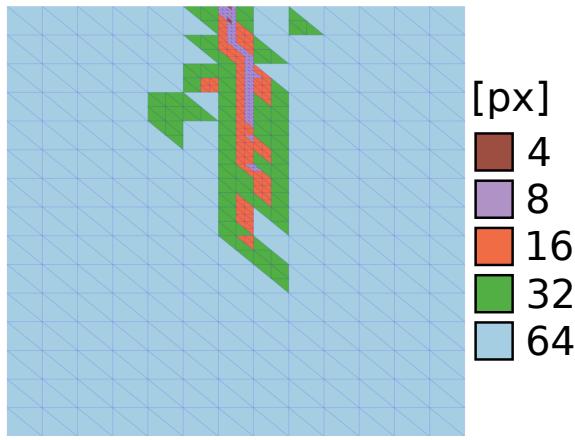

(b)

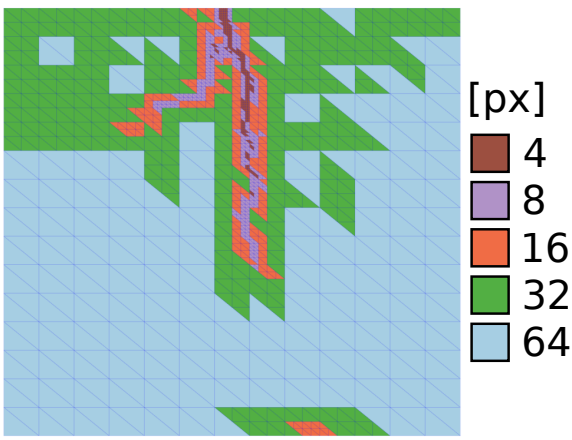

(d)

Figure 11: Element size field for different initial thresholds $\left(\llbracket u^{*} \rrbracket=k \sigma(\overline{\llbracket u \rrbracket})\right)$ for the frame \#30 of the WST case. (a) $k=12$, (b) $k=6$, (c) $k=3$, and (d) $k=1.5$

This observation is confirmed when analyzing the maps of MCOD reported in Figure 12. For the first two levels 


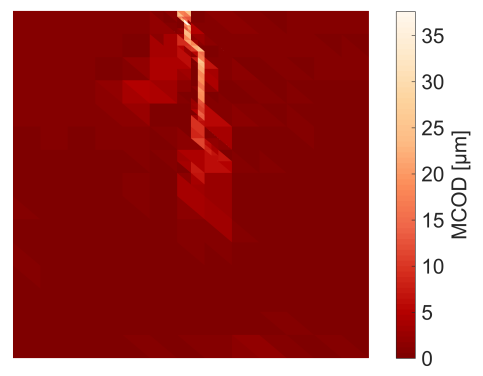

(a)

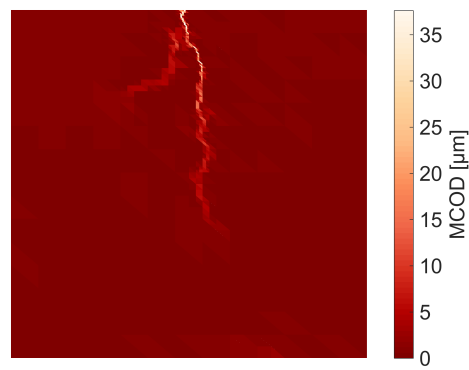

(c)

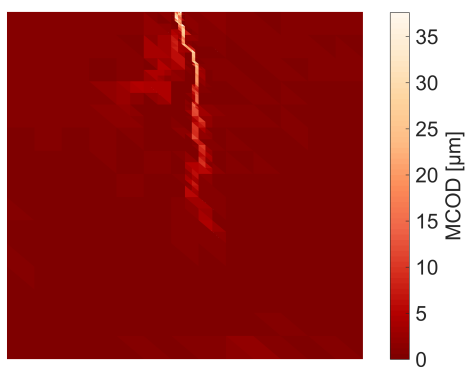

(b)

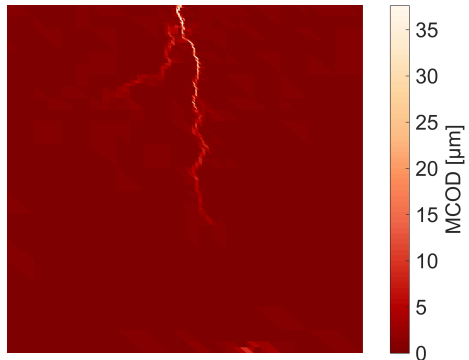

(d)

Figure 12: MCOD fields for different initial thresholds $\left(\llbracket u^{*} \rrbracket=k \sigma(\overline{\llbracket u \rrbracket})\right)$ for the frame \#30 of the WST case. (a) $k=12$, (b) $k=6$, (c) $k=3$, and (d) $k=1.5$

The acquisition noise in the GLR fields for the analyses using uniform meshes (Figure 9) is also observed when 239 using adaptive meshes (Figure 13). This result was expected because acquisition noise does not depend on the element 240 size. It is possible to depict the full developed crack, even on frame \#30, when the crack has not developed that far 241 (see Figure 9), due to residuals caused by mesh refinement. This result makes the use of GLR field difficult to analyze $\mathbf{2 4 2}$ crack propagation when using the proposed adaptive mesh, and reinforces the use of MCOD fields for this application. However, the residuals are concentrated around the cracked regions, and the localized refinement does not generate $\mathbf{2 4 4}$ fake cracks in the boundaries of domains with different element sizes (Figure 11). It is interesting to note that the crack 245 branches seen in the MCOD field are not visible in any of the GLR fields, thereby indicating that the corresponding $\mathbf{2 4 6}$ displacement levels remained very small (i.e., well below the pixel level). 


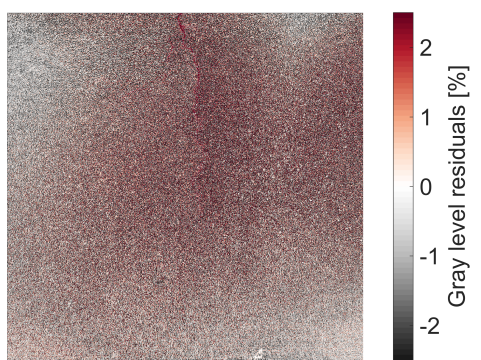

(a)

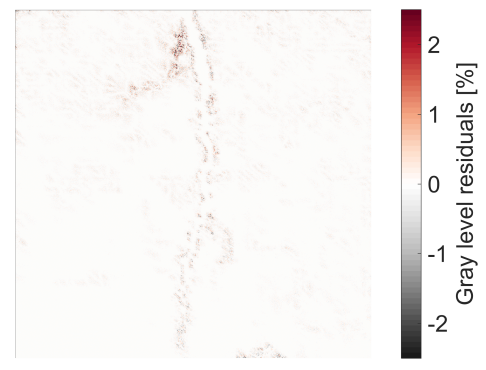

(c)

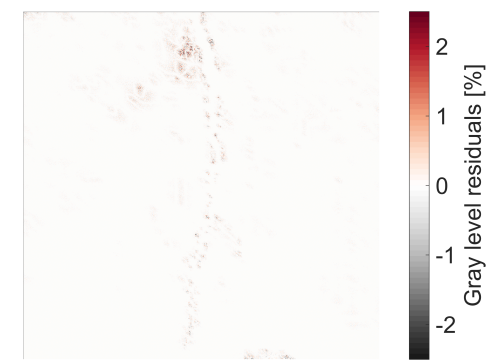

(b)

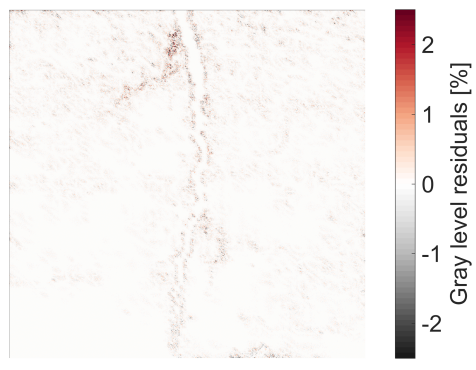

(d)

Figure 13: Gray level residual field for different initial thresholds $\left(\llbracket u^{*} \rrbracket=k \sigma(\overline{\llbracket u \rrbracket})\right)$ for the frame \# 30 of the WST case. (a) $k=12$, and the difference between two cases to depict the GLR caused by the refinement (b) $12-6$, (c) $12-3$, and (d) $12-1.5$

The normalized standard deviation GLR for each frame of the different adapted meshes are shown in Figure $14 \mathbf{2 4 8}$ and compared to the uniform mesh (designated as UM in the sequel) results from Figure 8. The standard deviations of 249 the GLR at the beginning of the experiment are virtually identical, showing that all adapted meshes perform the same $\mathbf{2 5 0}$ way, which is a further indication that no crack initiated. For frames closer to the end of the experiment, where the residuals due to crack openings are higher than those due to acquisition noise and lighting fluctuations, the AM with a threshold $k=3$ leads to GLR close to the UM 16 px discretization. The $k=1.5$ AM results are close to the 8 px UM, 253 even with this AM containing varying element sizes (Figure 11(c-d)). 


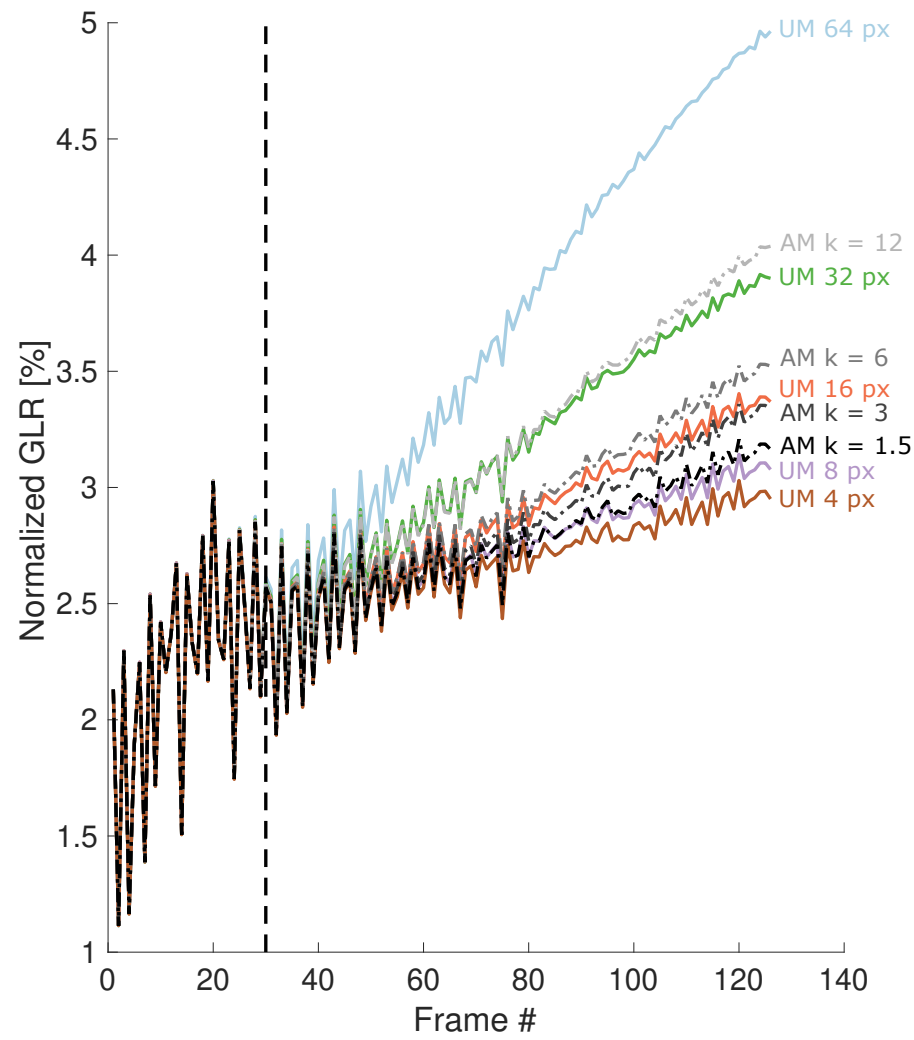

Figure 14: Comparison of the standard deviation of normalized gray level residuals field for the different discretizations (i.e., UM - solid lines, and AM - dash dotted lines). The vertical dashed black line marks frame \#30

The previous observations mean that the same quality of results is achieved using less degrees of freedom (Table 4). 255 The smallest standard deviation (STD) of the GLR for the last frame was found for the 4-px UM (i.e., it is equal to 256 2.95\%). Some 4 px elements are also present in AM for $k$ ranging from 6 down to 1.5 (Figure 11). The STD of GLR 257 levels are close to those of the 8-px UM, mainly for the AM with $k=1.5$. The size of the Hessians related to $k \leq 3$ are 258 6 and 4 times smaller than those of 4-px UM, respectively. This result indicates that the main source of GLR increase 259 is the lack of degrees of freedom in the cracked region. It demonstrates that a localized refinement can considerably $\mathbf{2 6 0}$ improve the description of cracks without huge increase in computational cost. Further, the computational cost is also 261 represented by the computation time (CT) for each analysis, which was obtained by summing the elapsed time during system solving in each iteration. Both CT and average iteration per image analyzed are shown in Table 4. 


\section{Table 4}

Sizes of the Hessian matrices for uniform (UM) and adapted (AM) meshes, computation time, average iterations per image pair, standard deviation of normalized gray level residuals for the series of 126 frames analyzed, and gain in AM Hessian size in comparison to UM with the same smallest element size. The column Feature contains the average element size $(\bar{L})$ and number of 4-px elements in brackets for AMs

\begin{tabular}{|c|c|c|c|c|c|c|}
\hline Type & Feature & Hessian size & $\mathrm{CT}[\mathrm{s}]$ & $\begin{array}{c}\text { Average iteration \# } \\
\text { per image pair }\end{array}$ & STD of GLR [\%] & Gain [\%] \\
\hline UM & $L=64 \mathrm{px}$ & 672 & 0.86 & 6 & 4.96 & - \\
\hline UM & $L=32 \mathrm{px}$ & 2,511 & 2.79 & 6 & 695 & - \\
\hline UM & $L=16 \mathrm{px}$ & 9,360 & 11.5 & 6 & 3.37 & - \\
\hline UM & $L=8 \mathrm{px}$ & 37,440 & 58 & 6 & 3.08 & - \\
\hline UM & $L=4 \mathrm{px}$ & 149,760 & 445.4 & 8 & 2.95 & - \\
\hline $\mathrm{AM}(k=12)$ & $\bar{L}=12 \mathrm{px}[2,592]$ & 10,002 & 108 & 7 & 4.04 & 93 \\
\hline $\mathrm{AM}(k=6)$ & $\bar{L}=11 \mathrm{px}[3,124]$ & 13,230 & 131 & 6 & 3.52 & 91 \\
\hline $\mathbf{A M}(\mathbf{k}=\mathbf{3})$ & $\bar{L}=10 \mathrm{px}[3,952]$ & $\mathbf{2 3 , 8 1 7}$ & 188.6 & 5 & 3.34 & 84 \\
\hline $\mathrm{AM}(k=1.5)$ & $\bar{L}=8 \mathrm{px}[6,348]$ & 37,668 & 371.5 & 5 & 3.16 & 75 \\
\hline
\end{tabular}

The AM with $k=3$ is the best compromise between a fine discretization and a low computational cost since the 264 refinement is concentrated in the whole cracked region (Figure 11 and Table 4). Higher $k$ values $(i . e$., $k=6,12)$ do $\mathbf{2 6 5}$ not refine enough the cracked region and do not contain 4-px elements that help to describe the crack path. Conversely, 266 when $k=1.5$, spots where there is no crack are refined. In terms of MCOD maps, the AM with $k=3$ leads to the same 267 type of resolution (Figure 12) as the 8-px UM (Figure 10) but with a smaller Hessian (see Table 4). Interestingly, even 268 if the conditioning of the system is degraded by the use of Lagrange multipliers, clearly visible in the CT of similar $\mathbf{2 6 9}$ Hessian sizes, the problem needed less iterations to converge due to the description of the crack region. Repeating $\mathbf{2 7 0}$ the uncertainty analyses using only the initial images and the AM with $k=3$ led to standard uncertainties for the 271 maximum eigen strain equal to $7.5 \times 10^{-5}$, and for the MCOD to $5 \times 10^{-2} \mu \mathrm{m}$ (see Figure 7 ).

One last question arises about subsequent crack propagation after frame \#30 since the mesh does not account for the fully propagated stage. Figure 15 summarizes the results after adapting the mesh for the very last frame using $k=3$. The element size distribution now has many 4 px elements concentrated along the crack path. Figure $15(\mathrm{~b}-\mathrm{c})$ reports the MCOD maps using this new AM, for the 30-th and the last frames, respectively. The fine discretization in 276 the whole crack path leads to noisier MCODs in earlier frames in regions that are probably not cracked yet (i.e., below the mid-height for frame \#30, see Figures 10 and 12). 
(a)

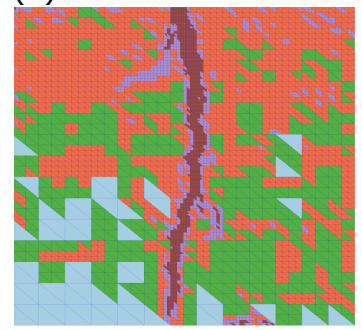

(b)

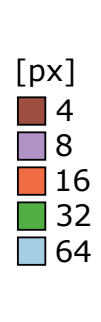

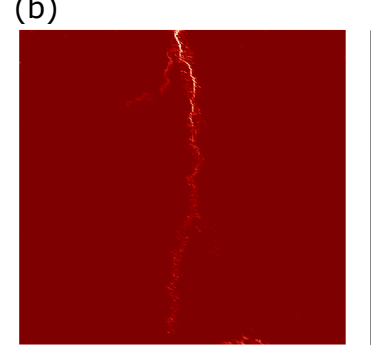

(c)

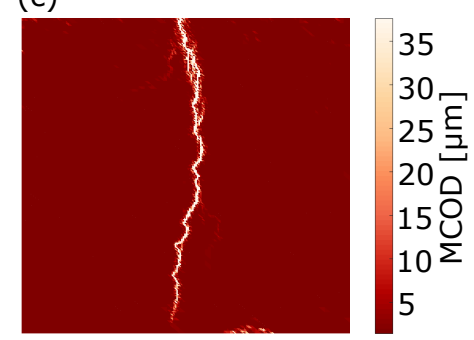

Figure 15: Results using a mesh adapted for the last frame. (a) Element size map. MCOD fields for the 30-th (b) and the last (c) frames

Figure 16 shows the GLR ratio between the first AM with $k=3$ and the one defined with the last frame. Both residuals have the same levels until frame \#30, and from that point on their ratio rises up to $17 \%$ since the first mesh did not account for crack propagation after this point. The AM at the end of the test shows noisier MCODs for earlier frames but with lower GLR. Such observations call for gradual mesh adaptions throughout the frames. However, more meshes (and consequently Hessian matrices) would need to be stored, and the benefits on the computational cost would need to be tested in future works.

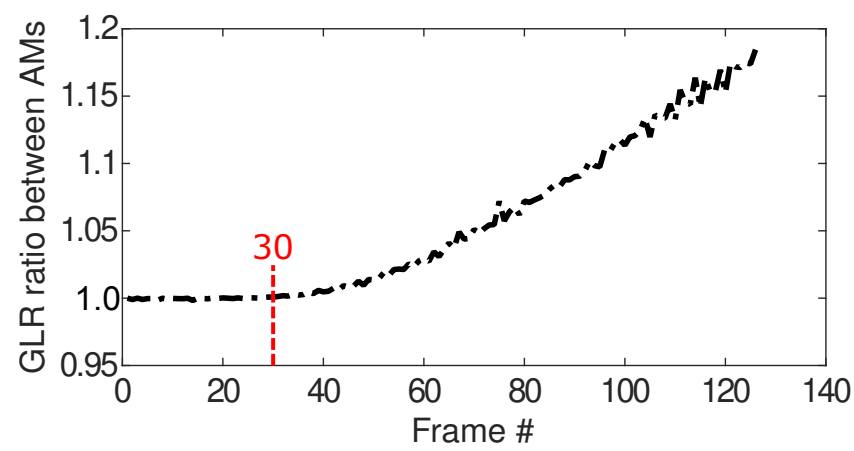

Figure 16: Ratio between the GLR for the AM for frame \#30-th and for the last one

\subsection{Damage due to MgO hydration in refractory castable (Case \#3)}

Magnesia $(\mathrm{MgO})$ is used in some refractory castable formulations [65]. $\mathrm{MgO}$ hydrates during curing and drying, 286 which may damage the material. In previous works, a climatic chamber was developed to evaluate such mechanism 287 using DIC [66]. A cubic specimen was kept in the climatic chamber at $50^{\circ} \mathrm{C}$ and $50 \%$ relative air humidity (Fig- 288 ure 17(a)). The acquired images were divided into two sets. First, 10 images with 10s interval among them were 289 acquired at the beginning of the experiment before crack initiation for uncertainty quantification. Second, 190 images 290 were acquired in intervals of $30 \mathrm{~min}$ starting just after the first set to evaluate damage. The procedure performed for the first experimental case is replicated hereafter for a crack network. The reference image and frame \#38 used for the 


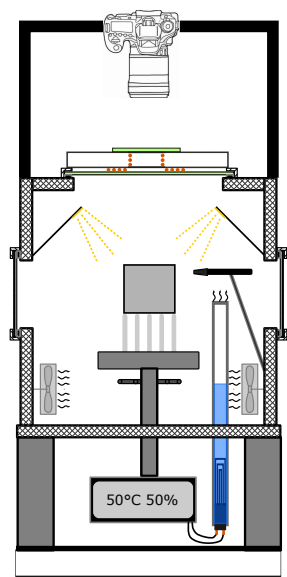

(a)

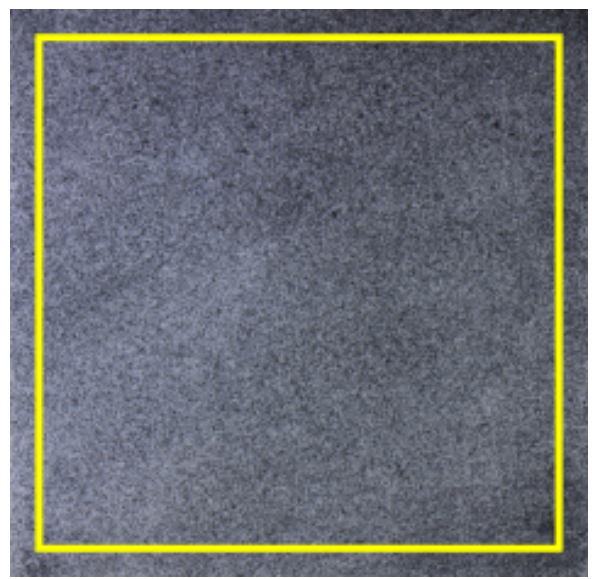

(b)

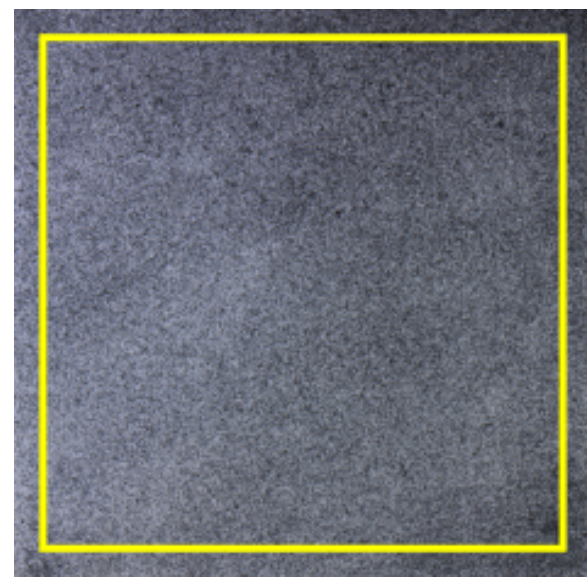

(c)

Figure 17: Schematic drawing of the climatic chamber with the refractory castable cube in the center (a), adapted from [66]. Reference image (b) and frame \#38 (c) for the $\mathrm{MgO}$ hydration experiment. The image sizes are $\approx 67 \times 67 \mathrm{~mm}^{2}$ $(\approx 1340 \times 1340 \mathrm{px})$ and the ROI (yellow square) size is $\approx 60 \times 60 \mathrm{~mm}^{2}(\approx 1200 \times 1200 \mathrm{px})$

The maximum eigen strain $\left(\epsilon_{1}\right)$ and MCOD uncertainties were calculated using the ten images acquired at the beginning of the experiment, before crack initiation. The temporal variance of $\epsilon_{1}$ and MCOD were computed for each 295 element, and their root means are shown in Figure 18 as functions of the inverse element size $L^{-1}$. The power $\alpha$ is now equal to -1.47 , which is smaller than the first experiment. It shows that uncertainty quantification should be performed for each test as the thresholds $\epsilon^{*}$ and $\llbracket u^{*} \rrbracket$ may vary from one experimental configuration to another one. The black stars in Figure 18 refer to the uncertainties computed for the optimal AM $(k=1.5)$ 


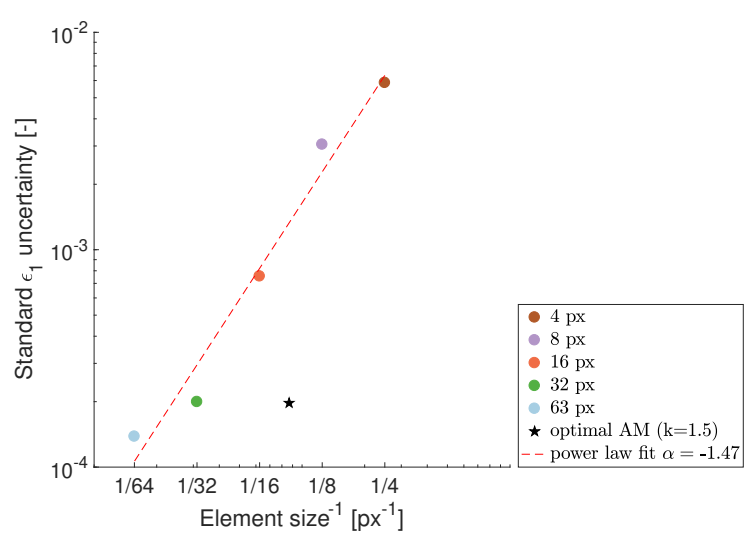

(a)

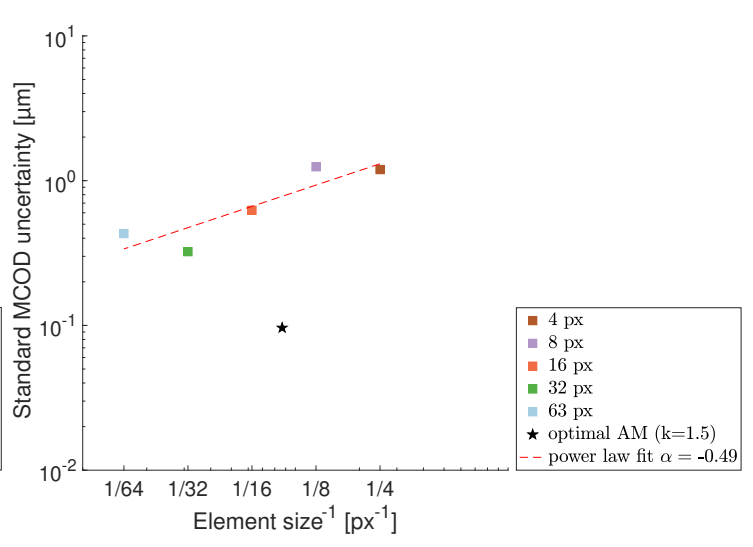

(b)

Figure 18: Standard uncertainties of the maximum eigen strain (a) and MCOD (b) as functions of the inverse of the element size $L$ for the initial images of the curing and drying experiment. The dashed lines depict power law fits according to Equations (24) and (25). The black stars mark the uncertainties for the optimal AM $(k=1.5)$

Figure 19 shows the standard deviation of the GLR when normalized by the dynamic range of the reference picture. 300 The effect of the element size for a uniform mesh (UM) is first investigated. At the beginning of the test, the overall GLR 301 level is mainly related to acquisition noise. The fluctuations are also due to lighting variations and the environment of $\mathbf{3 0 2}$ the climatic chamber. They are more pronounced in this second experiment, as highlighted by the spikes. Corrections $\mathbf{3 0 3}$ to the gray level fluctuations were suggested for such cases [67]. However, the correction was not used herein to $\mathbf{3 0 4}$ evidence the increase in noise sensitivity with a decrease of the element size (i.e., increase in spike amplitudes for small $\mathbf{3 0 5}$ elements). Further, the GLR for each UM are very close until frame \#25, for which cracks start to show measurable 306 MCOD. For the following frames, the GLR curves are distinguishable. It is caused by the lack of degrees of freedom (DOFs) to describe the crack network with coarse discretizations. This contribution to the GLR is less pronounced as 


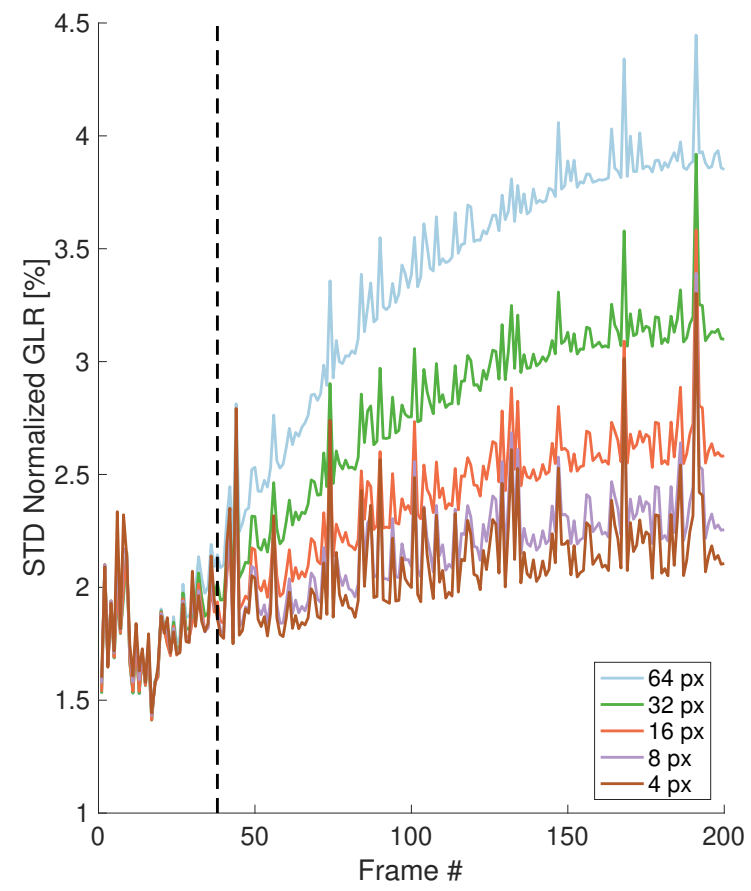

Figure 19: Normalized standard deviation of the gray level residuals (GLR) in each frame (as \% of the dynamic range) for uniform meshes with different element sizes. The vertical dashed line depicts frame \#38

The GLR fields for frame \#38 are shown in Figure 20. The field for the 64-px UM was set as a reference and 310 the GLR fields of the other UMs were subtracted from it to highlight their differences as in Case \#2 (see Figure 9). 311 Acquisition noise in the undamaged parts is identical for all UMs, and the different meshes sizes affect the regions 312 around the cracks as expected from Case \#2 results. The GLR caused by the crack network are thinner for UMs with 313 8- or 4-px elements. However, the 4-px UM was very sensitive to lighting changes (Figure 19), which hindered cracks 314 to be properly detected and quantified. Such observation makes the 8-px mesh a better choice. 

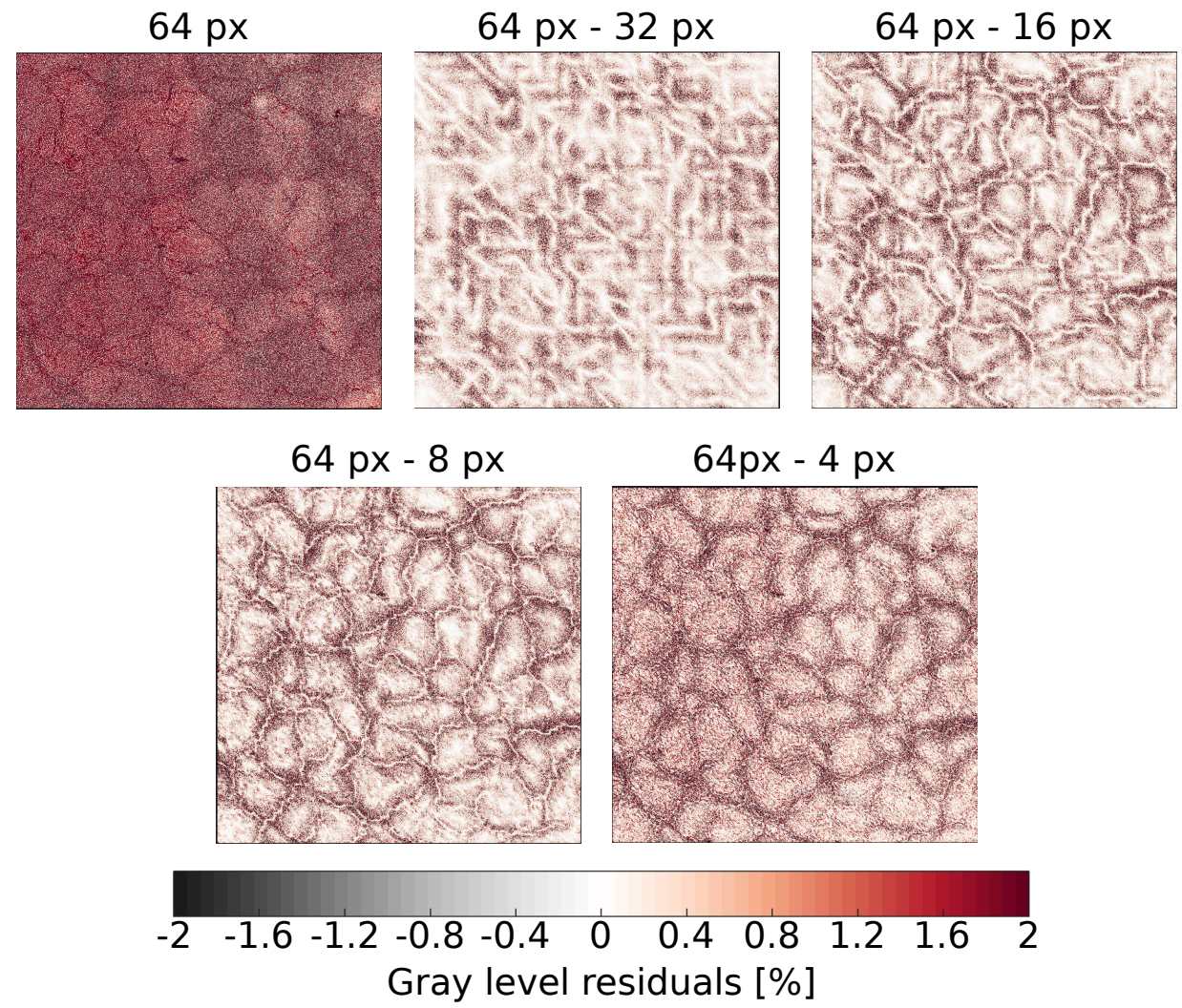

Figure 20: Normalized gray level residual fields for frame \#38 using UMs with different element sizes. For the sake of visualization the results from finer meshes were subtracted from the coarser mesh result (64 px). The color bar range was reduced to highlight the patterns in the fields

The corresponding MCOD fields are shown in Figure 21 for different UMs. The MCOD fields are less sensitive 316 to the acquisition noise than the GLR fields. The 63 and 32-px meshes yield very coarse crack patterns, and it is $\mathbf{3 1 7}$ difficult to identify a crack network from these results. For smaller element sizes (from 16 down to 4-px meshes), it is 318 easier to distinguish regions with high MCOD levels (i.e., cracks) and other ones with very low values (i.e., clusters 319 of aggregates). Further, the MCOD provided by the 4-px mesh showed smaller values, which indicates an effect of $\mathbf{3 2 0}$ mechanical regularization. 

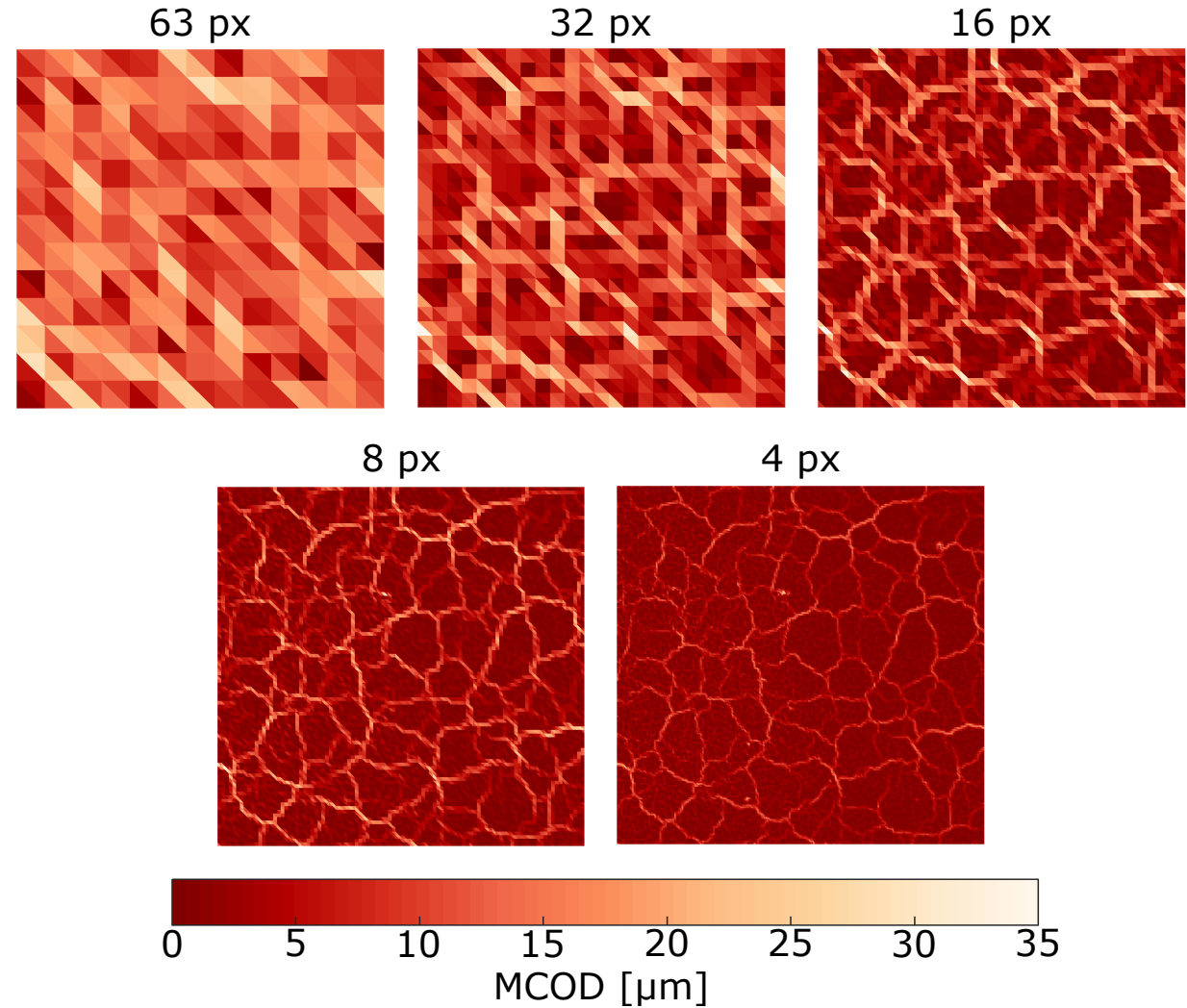

Figure 21: Mean crack opening displacement (MCOD) fields for frame \#38 when UMs with different element sizes are used $(1 \mathrm{px} \equiv 50 \mu \mathrm{m})$

Figure 22 shows the map of element sizes for four different values of $k$. For $k=12$ and 6 , the localized refinements are not very effective, and the AMs are similar to the UMs (Figure 22(a-b)). However, it is possible to depict clusters of same size as the elements (i.e., $16 \mathrm{px}$ and $32 \mathrm{px}$ ) for $k=3$ and 1.5 (Figure 22(c-d)). The case $k=1.5$ leads to the best crack network description with 8 px elements for the cracks and clusters of bigger sizes. It is worth noting that in the present case a very small level of $k$ was needed to properly capture all the fine details associated with a crack network. 


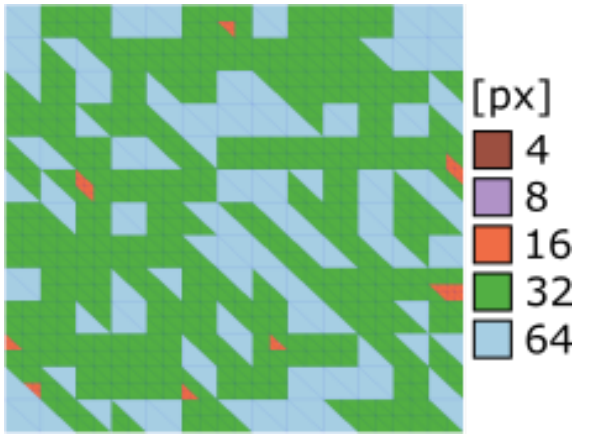

(a)

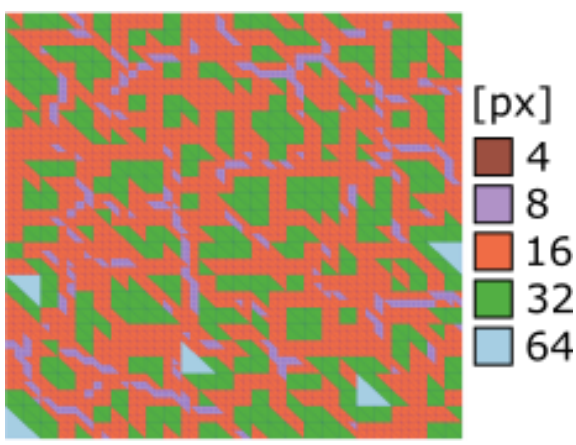

(c)

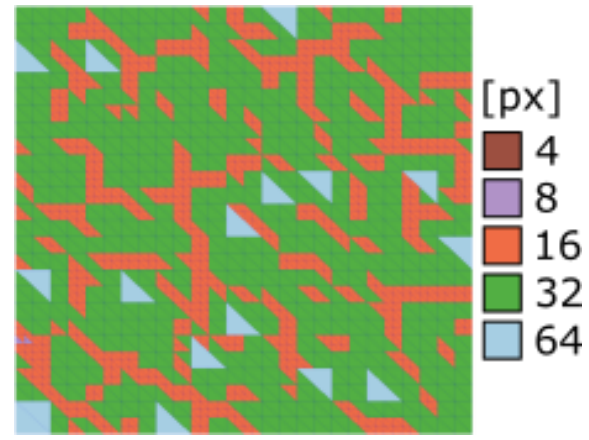

(b)

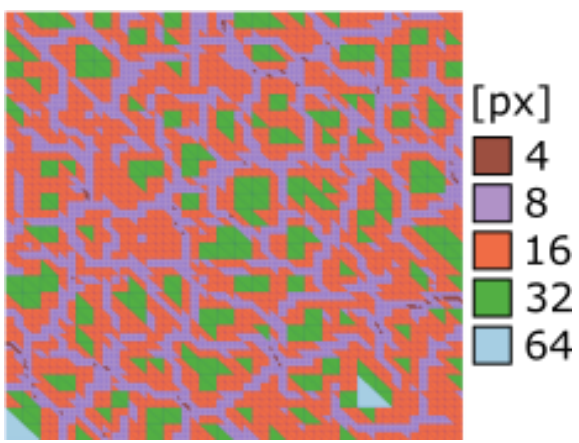

(d)

Figure 22: Element size field for different initial thresholds $\left(\llbracket u^{*} \rrbracket=k \sigma(\overline{\llbracket u \rrbracket})\right)$ for frame \#38 of the $\mathrm{MgO}$ hydration case. (a) $k=12$, (b) $k=6$, (c) $k=3$, and (d) $k=1.5$

The resultant MCOD fields for the different AMs are shown in Figure 23 for frame \#38. The fields for $k=1.5328$ AM (Figure 23(d)) and 8-px UM (Figure 21) are very similar. The crack network is "blurred" with an increase of $k, 329$ and follows a similar trend as augmenting the element size. This similarity also indicates that $k=1.5$ leads to the best 330 AM for Case \#3. 


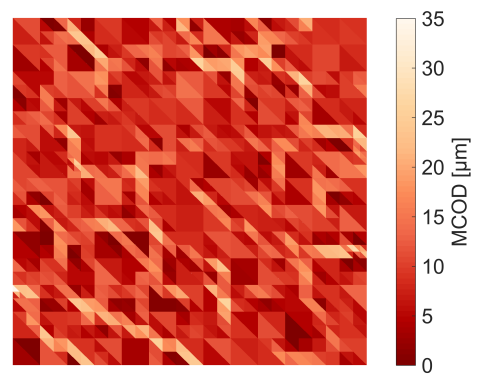

(a)

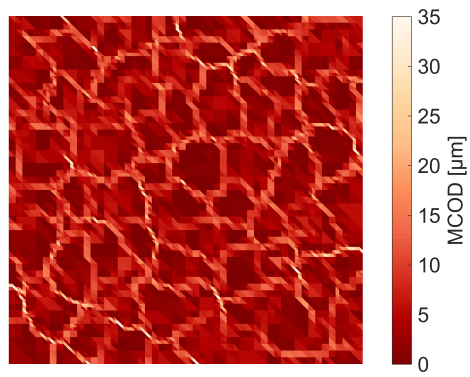

(c)

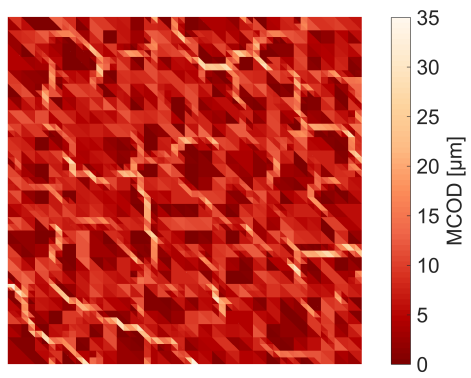

(b)

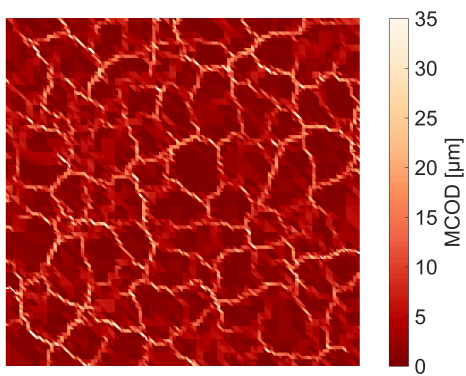

(d)

Figure 23: MCOD fields for picture \#38 and different initial thresholds $\left(\llbracket u^{*} \rrbracket=k \sigma(\overline{\llbracket u \rrbracket})\right)$ of the $\mathrm{MgO}$ hydration case. (a) $k=12$, (b) $k=6$, (c) $k=3$, and (d) $k=1.5$

The corresponding GLR fields are shown in Figure 24 for the studied thresholds $k$. The acquisition noise remains 332 present in all cases since it is not dependent on the element size. Similarly to the previous results, the crack pattern is $\mathbf{3 3 3}$ more visible in the GLR maps of higher thresholds due to interpolation errors related to fewer DOFs. 


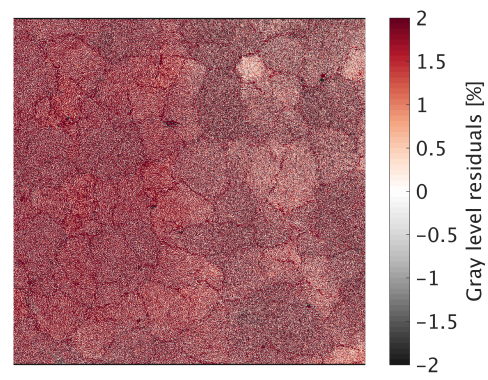

(a)

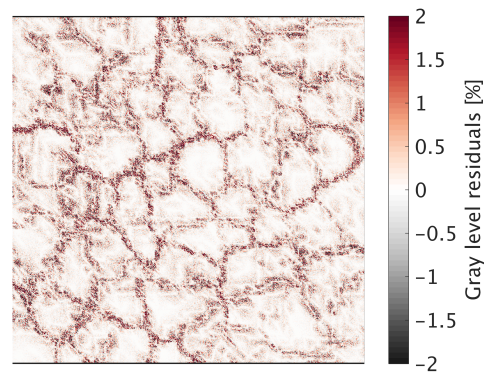

(c)

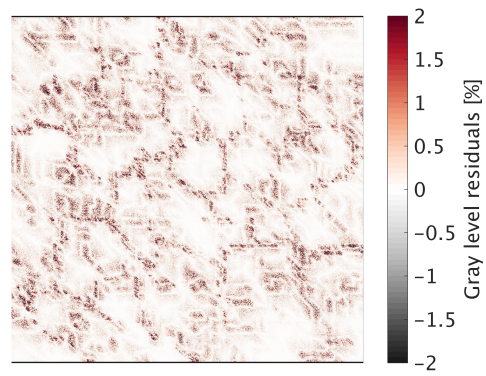

(b)

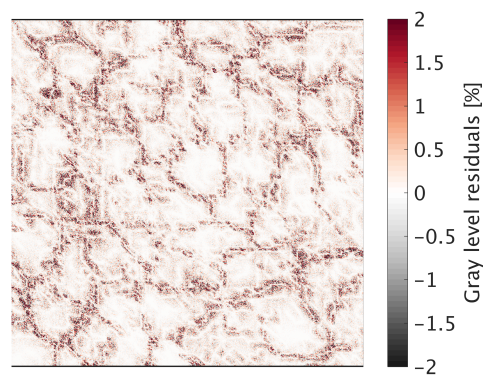

(d)

Figure 24: Gray level residual field for different initial thresholds $\left(\llbracket u^{*} \rrbracket=k \sigma(\overline{\llbracket \rrbracket})\right)$ for the frame \# 38 of the WST case. (a) $k=12$, and difference between two of them to depict the GLR caused by the refinement (b) $12-6$, (c) $12-3$, and (d) $12-1.5$

The normalized standard deviation of GLR comparing UMs and AMs is shown in Figure 25 for the whole exper- 335 iment. As before, the smallest residuals are observed for the 4 px UM, and AM with $k=3$ and 1.5, which are close 336 to 16 px and 8 px UMs, respectively. The similarity of the curves before frame \#38 further evidences that no (or very $\mathbf{3 3 7}$ little) cracks propagated until this point. From frame \#38 onward, higher residuals are related to higher gray levels 338 induced by the newly created cracks, and consequently the normalized GLR follows the amount of DOFs to allow for 339 such complex kinematics associated with the crack network to be captured. An additional contribution is given by the $\mathbf{3 4 0}$ fact that mesh adaption was not performed again. 


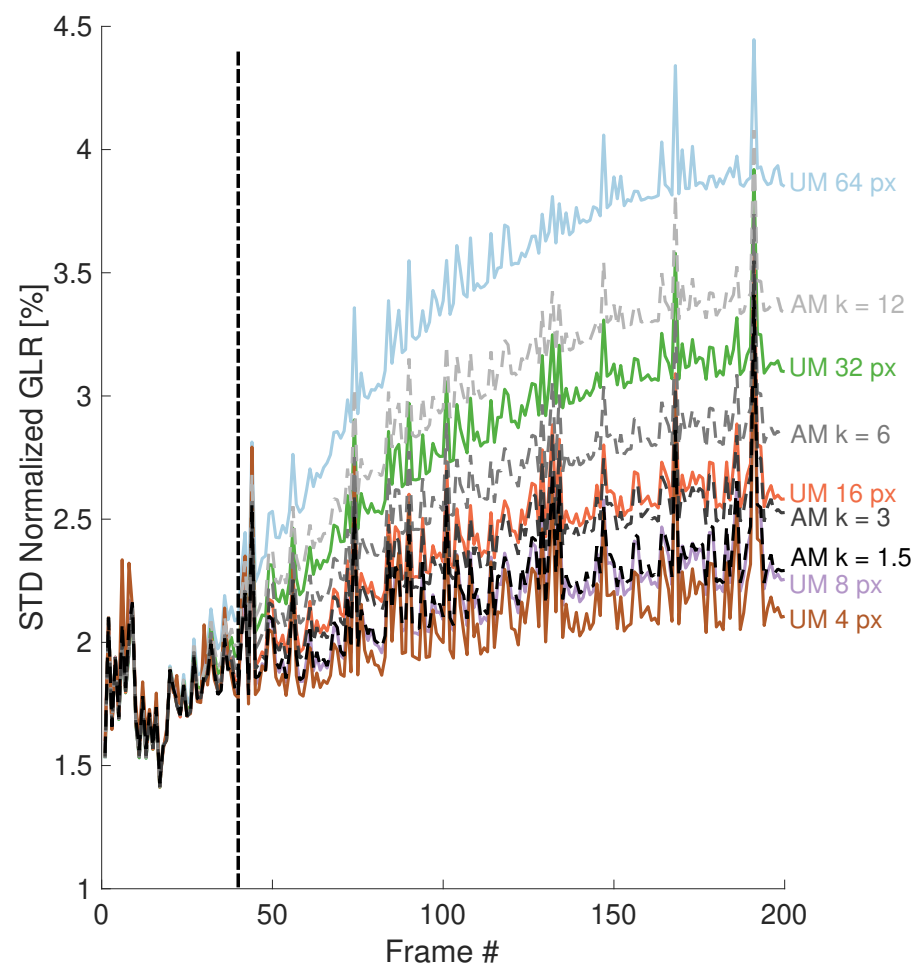

Figure 25: Standard deviation of GLR for the different discretizations (i.e., UM: solid lines, and AM: dash dotted lines).

The vertical dashed line marks frame \#38

The Hessian size for each case shown in Figure 25 is reported in Table 5 along with the normalized GLR for the 342 last frame, as well as the computation time (CT), and the average iteration number. As pointed out for the numerical $\mathbf{3 4 3}$ example, the benefits of using AMs are inversely proportional to the crack density. Further, the local refinement of 344 AMs did not reduce the average iteration number in Case \#3 as was noted in Case \#2. The main benefit comes from $\mathbf{3 4 5}$ using the AM with $k=1.5$, with similar GLR and Hessian size to the 8 px UM but containing few 4 px elements. The 346 uncertainty analyses using only the initial images and AM with $k=1.5$ led to standard uncertainties for the maximum $\mathbf{3 4 7}$ eigen strain and MCOD equal to $2 \times 10^{-4}$ and $0.9 \mu \mathrm{m}$, respectively (see Figure 18). 


\section{Table 5}

Sizes of the Hessian matrices for uniform (UM) and adapted (AM) meshes, computation time, average iterations per image pair, standard deviation of normalized gray level residuals for the series of 200 frames analyzed, and gain in AM Hessian size in comparison to UM with the same smallest element size. The column Feature contains the average element size $(\bar{L})$ and number of 4-px elements in brackets for AMs

\begin{tabular}{|c|c|c|c|c|c|c|}
\hline Type & Feature & Hessian size & $\mathrm{CT}[\mathrm{s}]$ & $\begin{array}{c}\text { Average iteration \# } \\
\text { per image pair }\end{array}$ & STD of GLR [\%] & Gain [\%] \\
\hline UM & $L=64 \mathrm{px}$ & 588 & 0.9 & 4.6 & 3.85 & - \\
\hline UM & $L=32 \mathrm{px}$ & 2,028 & 5.18 & 4.7 & 3.10 & - \\
\hline UM & $L=16 \mathrm{px}$ & 7,500 & 15.3 & 4.8 & 2.58 & - \\
\hline UM & $L=8 \mathrm{px}$ & 30,000 & 64.6 & 5.0 & 2.26 & - \\
\hline UM & $L=4 \mathrm{px}$ & 120,000 & 1677 & 23.5 & 2.11 & - \\
\hline $\operatorname{AM}(k=12)$ & $\bar{L}=34 \mathrm{px}[0]$ & 3,078 & 12.72 & 4.6 & 3.34 & 59 \\
\hline $\operatorname{AM}(k=6)$ & $\bar{L}=21 \mathrm{px}[0]$ & 7,803 & 34 & 4.6 & 2.83 & 74 \\
\hline $\operatorname{AM}(k=3)$ & $\bar{L}=14 \mathrm{px}[0]$ & 16,845 & 87.6 & 4.7 & 2.53 & 44 \\
\hline $\operatorname{AM}(k=1.5)$ & $\bar{L}=10 \mathrm{px}[312]$ & 31,422 & 356.6 & 4.7 & 2.29 & 74 \\
\hline
\end{tabular}

As in the previous case, one last point of interest arises from the frame used for mesh adaption. Figure 26 shows the results of an AM with $k=1.5$ for the very last frame of the test. The crack path is finely described with very small $\mathbf{3 5 0}$ elements (Figure 26(a)), with considerably more 4-px elements than for the 38-th frame (Figure 22). The corresponding 351 MCOD fields are compared in Figure 26(b-c). A similar trend (to the WST case) of noisier results for the earlier frame, 352 and a cleaner description for the last frame. The fluctuations seen for frame \#38 are due the fine discretization related $\mathbf{3 5 3}$ to crack openings and not its initiation. Although the crack network was already visible in earlier frames, its openings $\mathbf{3 5 4}$ were very small.

(a)

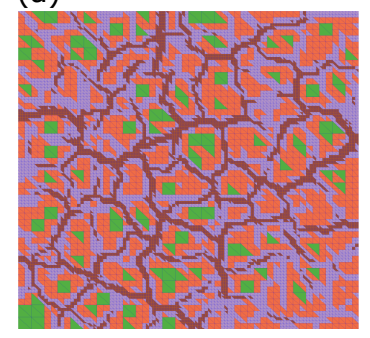

(b)

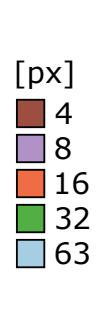

\section{(b)}

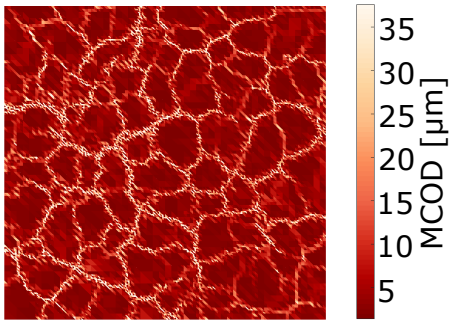

(c)

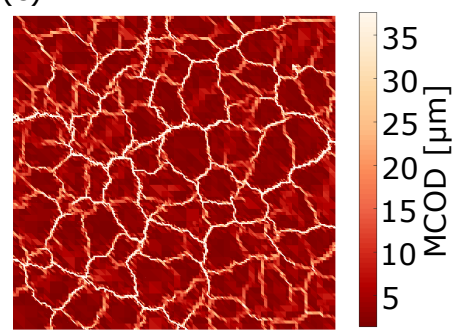

Figure 26: Results using a mesh adapted for the last frame. (a) Element size map. MCOD fields for the 38-th (b) and the last (c) frames 
Last, the ratio between GLR for the AM for the 38-th frame and the last picture is shown in Figure 27. For earlier 356 frames, a coarser discretization (provided by the 38-th frame) was slightly better, and as cracks subsequently opened, 357 the second mesh provided little gain. This observation is further evidence that the crack network was already formed $\mathbf{3 5 8}$ in early frames, which is different from the WST case in which propagation occurred during the whole experiment.

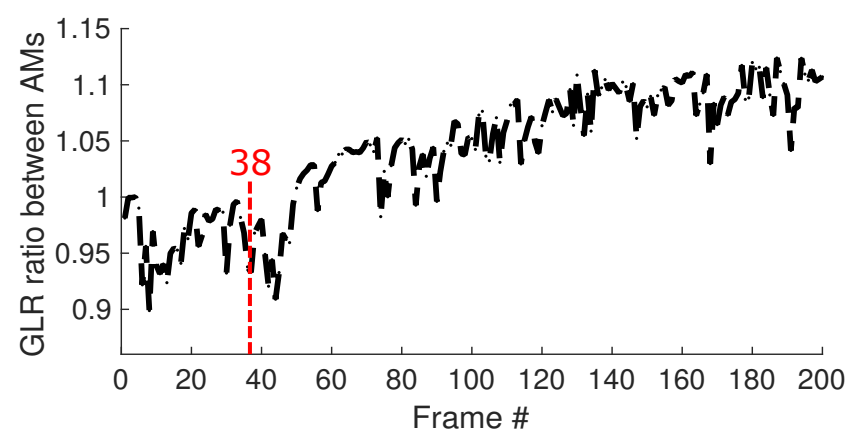

Figure 27: Ratio between the GLR for the AM for frame \#38-th and for the last one

\section{Conclusion}

A procedure to locally refine meshes for Digital Image Correlation (DIC) analyses was proposed. It is referred $\mathbf{3 6 1}$ to as adaptive meshing as it is guided by auxiliary fields, i.e., gray level residuals (artificial case) or maximum eigen $\mathbf{3 6 2}$ strain (experimental cases). At each refinement iteration, the selected elements were divided into four new ones by $\mathbf{3 6 3}$ splitting the edges into two new ones, which created hanging nodes (i.e., nodes with a lack of connectivity). Lagrange 364 multipliers reconnected these nodes to the mesh, ensuring that the displacement of each hanging node be the mean of its 365 parent nodes. This methodology was developed to improve the meshing process for DIC analyses of cracked materials with efficient computational cost, and with at least similar accuracy compared to fine uniform meshes. Although $\mathbf{3 6 7}$ the discussion mainly focused on cracks, other auxiliary fields related to localized phenomena (e.g., phase transition, $\mathbf{3 6 8}$ plasticity) could be used.

The artificial test case illustrated the relationship between the number of cracks and the gain in applying AM 370 procedures. The refinement criterion was based on the gray levels at the pixel scale. It was demonstrated that the gains of AM were inversely proportional to the surface crack density (i.e., higher for single crack analysis and decayed as the number and crack extensions increased). In other words, the studied phenomenon (i.e., cracks) passed from localized to diffuse states.

In the investigated experimental cases, AM was first applied to a Wedge Splitting Test (WST), since a low crack 375 density was expected and highlighted the benefits of the proposed procedure. Five different Uniform Meshes (UMs) 
demonstrated that AMs reduced memory storage by decreasing the DIC Hessian size while keeping similar descriptions 378 of the crack kinematics.

The second experimental case was devoted to crack network formation due to the hydration of an $\mathrm{MgO}$ castable, 380 with a considerably higher crack density. This case was also related to higher uncertainty levels since the experiment $\mathbf{3 8 1}$ was performed inside a climatic chamber with additional artifacts (i.e., glass windows and humidity/temperature of 382 the environment). Even in this challenging case, the AM procedure showed benefits for reducing the memory load $\mathbf{3 8 3}$ with a smaller Hessian matrix. An additional benefit for this case was that the map for the element size after adapting 384 the mesh already showed the overall crack network. Even if the artificial example pointed out that the benefits would $\mathbf{3 8 5}$ be smaller for high crack densities, the experimental cases proved that it was indeed more efficient for smaller crack $\mathbf{3 8 6}$ densities but it could also be efficient for dense crack networks when a very fine description was sought.

The criterion to divide the elements was based on the standard uncertainty of the Mean Crack Opening Displace- 388 ment (MCOD) per element. This metric was estimated by analyzing the initial frames of each experiment, where no 389 crack initiation was expected, and using uniform meshes with the same element sizes as those that will make part of the adapted meshes. It was shown that the uncertainties were different for both experiments (because of differences in 391 experimental environment), which further validated the choice of uncertainty quantifications. The final threshold was proportional to the MCOD uncertainty to only account for cracks (i.e., a multiplicative factor $k>1$ was considered). Different values of $k$ were chosen for the two investigated cases, which indicates that $k$ may depend on the surface crack density. Additional investigation are needed to confirm such observation.

Although the numerical test case underscored that the methodology would be best suited for single cracks, it was not straightforward for the experimental cases. Even though both cases benefited from an adapted mesh, the crack network had one additional advantage since crack initiations showed the full crack path from early frames on, meaning that the mesh could be adapted for different frames with similar results. For the WST, with one macrocrack and few branches, the crack path changed throughout the experiment and further studies should be conducted to find a suitable temporal mesh adaptation strategy to describe gradual crack propagation (e.g., using developing meshes, damaging 401 elements in the cracked region or using node splitting techniques).

Last, the AM procedure has a high potential of reducing the computational cost of Digital Volume Correlation 403 analysis, where data are usually considerably bigger than DIC, especially for cases with localized phenomena. Implementing a refinement termination based on the gray level residuals, and also, real-time applications of this procedure 405 during the DIC analyses of many images, are other future improvements of this new methodology. 


\section{Acknowledgments}

This study was financed in part by the Coordenação de Aperfeiçoamento de Pessoal de Nível Superior - Brasil 408 (CAPES) - Finance Code 001, and the support by the PDSE grant \#8881.188511/2018-01 used during the internship 409 of VS at LMT, CAPES (Brazil) and \#2010/20920-9, \#2018/02801-4, \#2018/15266-0 and \#2018/23081-0, São Paulo 410 Research Foundation (FAPESP). The authors would like to thank D.B.D. Stafuzza for his help in performing the WST 411 experiment.

\section{Credit authorship statement}

V.F. Sciuti: Conceptualization, Methodology, Investigation, Software, Original draft preparation, Writing - Review

R. Vargas: Conceptualization, Methodology, Investigation, Software, Original draft preparation, Writing - Review

R.B. Canto: Supervision, Conceptualization, Writing - Review Editing, Resources, Funding acquisition, Project administration

F. Hild: Supervision, Conceptualization, Methodology, Software, Formal analysis, Writing - Review Editing, 420 Resources, Funding acquisition, Project administration

\section{Declaration of Competing Interest}

The authors declare that they have no known competing financial interests or personal relationships that could have

\section{References}

[1] M. Sutton, W. Wolters, W. Peters, W. Ranson, S. McNeill, Determination of displacements using an improved digital correlation method, Im. Vis. Comp. 1 (3) (1983) 133-139.

[2] T. Chu, W. Ranson, M. Sutton, W. Peters, Applications of digital-image-correlation techniques to experimental mechanics, Exp. Mech. 3 (25) (1985) 232-244.

[3] M. Sutton, Computer vision-based, noncontacting deformation measurements in mechanics: A generational transformation, Appl. Mech. Rev. 65 (AMR-13-1009) (2013) 050802.

4] M. Sutton, S. McNeill, J. Helm, Y. Chao, Advances in Two-Dimensional and Three-Dimensional Computer Vision, Vol. Topics in Appl. Phys.,

[5] M. Sutton, J. Orteu, H. Schreier, Image correlation for shape, motion and deformation measurements: Basic Concepts, Theory and Applications, Springer, New York, NY (USA), 2009.

[6] F. Hild, S. Roux, Digital image correlation, in: P. Rastogi, E. Hack (Eds.), Optical Methods for Solid Mechanics. A Full-Field Approach, Wiley-VCH, Weinheim (Germany), 2012, pp. 183-228. 
[7] W. Peters, W. Ranson, J. Kalthoff, S. Winkler, A study of dynamic near-crack-tip fracture parameters by digital image analysis, J. Phys. Coll. 438 46 (C5) (1985) 631-638.

[8] S. McNeill, W. Peters, M. Sutton, Estimation of stress intensity factor by digital image correlation, Eng. Fract. Mech. 28 (1) (1987) 101-112.

[9] S. Roux, J. Réthoré, F. Hild, Digital image correlation and fracture: An advanced technique for estimating stress intensity factors of 2d and 3d cracks, J. Phys. D: Appl. Phys. 42 (2009) 214004.

[10] F. Mathieu, P. Aimedieu, J. Guimard, F. Hild, Identication of interlaminar fracture properties of a composite laminate using local full-field kinematic measurements and finite element simulations, Comp. Part A 49 (2013) 203-213.

[11] J. Abanto-Bueno, J. Lambros, Experimental determination of cohesive failure properties of a photodegradable copolymer, Exp. Mech. 45 (2) (2005) $144-152$.

[12] R. Fedele, B. Raka, F. Hild, S. Roux, Identification of adhesive properties in glare assemblies by digital image correlation, J. Mech. Phys. Solids 57 (2009) 1003-1016.

3] J. Réthoré, R. Estevez, Identification of a cohesive zone model from digital images at the micron-scale, J. Mech. Phys. Solids 61 (6) (2013) $1407-1420$.

[14] S. Roux, F. Hild, Stress intensity factor measurements from digital image correlation: post-processing and integrated approaches, Int. J. Fract. $140(1-4)(2006)$ 141-157.

[15] M. Williams, On the stress distribution at the base of a stationary crack, ASME J. Appl. Mech. 24 (1957) 109-114.

[16] G. Broggiato, Adaptive image correlation technique for full-field strain measurement, in: C. Pappalettere (Ed.), 12th Int. Conf. Exp. Mech., McGraw Hill, Lilan (Italy), 2004, pp. 420-421.

[17] Y. Sun, J. Pang, C. Wong, F. Su, Finite-element formulation for a digital image correlation method, Appl. Optics 44 (34) (2005) $7357-7363$.

[18] G. Besnard, F. Hild, S. Roux, "Finite-element" displacement fields analysis from digital images: Application to Portevin-Le Chatelier bands, Exp. Mech. 46 (2006) 789-803.

19] N. Moës, J. Dolbow, T. Belytschko, A finite element method for crack growth without remeshing, Int. J. Num. Meth. Eng. 46 (1) (1999) $133-150$.

[20] J. Réthoré, S. Roux, F. Hild, From pictures to extended finite elements: Extended digital image correlation (X-DIC), C. R. Mécanique 335 (2007) 131-137.

[21] J. Réthoré, F. Hild, S. Roux, Extended digital image correlation with crack shape optimization, Int. J. Num. Meth. Eng. 73 (2) (2008) $248-272$.

[22] J. Réthoré, J. Tinnes, S. Roux, J. Buffière, F. Hild, Extended three-dimensional digital image correlation (X3D-DIC), C. R. Mécanique 336 (2008) 643-649.

23] J. Rannou, N. Limodin, J. Réthoré, A. Gravouil, W. Ludwig, M. Baïetto, J. Buffière, A. Combescure, F. Hild, S. Roux, Three dimensional experimental and numerical multiscale analysis of a fatigue crack, Comp. Meth. Appl. Mech. Eng. 199 (2010) 1307-1325.

4] S. Roux, F. Hild, H. Leclerc, Mechanical assistance to DIC, in: H. Espinosa, F. Hild (Eds.), Full field measurements and identification in Solid Mechanics, Vol. Procedia IUTAM, 4, Elsevier, 2012, pp. 159-168.

$25]$ E. Fagerholt, E. Østby, T. Børvik, O. Hopperstad, Investigation of fracture in small-scale SENT tests of a welded X80 pipeline steel using Digital Image Correlation with node splitting, Engineering Fracture Mechanics 96 (2012) 276-293.

26] E. Fagerholt, T. Børvik, O. S. Hopperstad, Measuring discontinuous displacement fields in cracked specimens using digital image correlation with mesh adaptation and crack-path optimization, Optics Lasers Eng. 51 (3) (2013) 299-310.

[27] R. Vargas, A. Tsitova, F. Bernachy-Barbe, B. Bary, R. Canto, F. Hild, On the identification and validation of fracture mechanics models for curved crack in mortar, Strain (2020). 
[28] J. Réthoré, F. Hild, S. Roux, Shear-band capturing using a multiscale extended digital image correlation technique, Comp. Meth. Appl. Mech.

Eng. 196 (49-52) (2007) 5016-5030.

[29] R. Fedele, L. Galantucci, A. Ciani, Global 2D digital image correlation for motion estimation in a finite element framework: a variational formulation and a regularized, pyramidal, multi-grid implementation, Int. J. Num. Meth. Eng. 96 (12) (2013) 739-762.

[30] M. Bornert, F. Brémand, P. Doumalin, J. Dupré, M. Fazzini, M. Grédiac, F. Hild, S. Mistou, J. Molimard, J. Orteu, L. Robert, Y. Surrel,
P. Vacher, B. Wattrisse, Assessment of digital image correlation measurement errors: Methodology and results, Exp. Mech. 49 (3) (2009)

[30] M. Bornert, F. Brémand, P. Doumalin, J. Dupré, M. Fazzini, M. Grédiac, F. Hild, S. Mistou, J. Molimard, J. Orteu, L. Robert, Y. Surrel,
P. Vacher, B. Wattrisse, Assessment of digital image correlation measurement errors: Methodology and results, Exp. Mech. 49 (3) (2009) $353-370$.

[31] X. Wang, S. Ma, Mesh-Based Digital Image Correlation Method Using Non-Uniform Elements for Measuring Displacement Fields with High
Gradient, Experimental Mechanics 54 (2014) 1545-1554.

[31] X. Wang, S. Ma, Mesh-Based Digital Image Correlation Method Using Non-Uniform Elements for Measuring Displacement Fields with High
Gradient, Experimental Mechanics 54 (2014) 1545-1554.

[32] A. Baldi, F. Bertolino, Assessment of h-refinement procedure for global digital image correlation, Meccanica 51 (2016) $979-991$.

[33] S. Panin, V. Titkov, P. Lyubutin, Effect of the mesh size of the vector displacement field on the strain estimate in the digital image correlation method, Journal of Applied Mechanics and Technical Physics 58 (2017) 425-435.

[34] F. Hild, A. Bouterf, S. Roux, Damage measurements via DIC, International Journal of Fracture 191 (1-2) (2015) $77-105$.

[35] S. Ma, Z. Zhao, X. Wang, Mesh-based digital image correlation method using higher order isoparametric elements, J. Strain Analysis 47 (3) (2012) 163-175.

[36] X. Li, G. Fang, J. Zhao, Z. Zhang, X. Wu, Local Hermite (LH) Method: An accurate and robust smooth technique for high-gradient strain reconstruction in digital image correlation, Optics and Lasers in Engineering 112 (2019) 26-38.

[37] L. Wittevrongel, P. Lava, S. Lomov, D. Debruyne, A self adaptive global digital image correlation algorithm, Experimental Mechanics 55 (2014) 361-378.

[38] L. Wittevrongel, P. Lava, S. V. Lomov, D. Debruyne, $C^{n}$-continuity in Digital Image Correlation: Implementation and Validation of $C^{-1}, C^{0}$ and $C^{1}$ Algorithms, Strain 51 (6) (2015) 444-458.

[39] S. Kleinendorst, J. Hoefnagels, C. Verhoosel, A. Ruybalid, On the use of adaptive refinement in isogeometric digitalimage correlation, International Journal for Numerical Methods in Engineering 104 (10) (2015) 944-962.

[40] B. Wagne, S. Roux, F. Hild, Spectral approach to displacement evaluation from image analysis, Eur. Phys. J. AP 17 (2002) $247-252$.

[41] H. Leclerc, J. Périé, S. Roux, F. Hild, Integrated digital image correlation for the identification of mechanical properties, Vol. LNCS 5496, Springer, Berlin (Germany), 2009, pp. 161-171.

2] J. Réthoré, S. Roux, F. Hild, An extended and integrated digital image correlation technique applied to the analysis of fractured samples: The equilibrium gap method as a mechanical filter, European Journal of Computational Mechanics 18 (3-4) (2009) $285-306$.

[43] Z. Tomičevć, F. Hild, S. Roux, Mechanics-aided digital image correlation, The Journal of Strain Analysis for Engineering Design 48 (5) (2013) 330-343.

4] Claire, F. Hild, S. Roux, A finite element formulation to identify damage fields: The equilibrium gap method, Int. J. Num. Meth. Engng.

[45] A. Mendoza, J. Neggers, F. Hild, S. Roux, Complete mechanical regularization applied to digital image and volume correlation, Computer Methods in Applied Mechanics and Engineering 355 (2019) 27-43.

[46] H. Leclerc, J. Périé, S. Roux, F. Hild, Voxel-scale digital volume correlation, Exp. Mech. 51 (4) (2011) 479-490.

[47] A. Vakulenko, M. Kachanov, Continuum theory of medium with cracks, Isv. AN SSSR, Mekh. Tverdogo Tela 4 (1971) 159-166.

[48] F. Hild, S. Roux, Comparison of local and global approaches to digital image correlation, Experimental Mechanics 52 (9) (2012) 1503-1519.

[49] H. Leclerc, J. Neggers, F. Mathieu, S. Roux, F. Hild, Correli 3.0, IDDN.FR.001.520008.000.S.P.2015.000.31500 (2015). 
[50] E. Tschegg, Prüfeinrichtung zur Ermittlung von bruchmechanishen Kennwerten sowie hiefür geeignete, Prüfkörper, Austrian Pat. AT 390328B, 514 registered (1986).

[51] H. Harmuth, Stability of crack propagation associated with fracture energy determined by wedge splitting specimen, Theoretical and Applied $\mathbf{5 1 6}$ Fracture Mechanics 23 (1995) 103-108.

[52] Y. Belrhiti, O. Pop, A. Germaneau, P. Doumalin, J. C. Dupré, H. Harmuth, M. Huger, T. Chotard, Investigation of the impact of micro-cracks on fracture behavior of magnesia products using wedge splitting test and digital image correlation, Journal of the European Ceramic Society 35 (2) (2015) 823-829.

53] Y. Dai, D. Gruber, H. Harmuth, Determination of the fracture behaviour of MgO-refractories using multi-cycle wedge splitting test and digital image correlation, Journal of the European Ceramic Society 37 (15) (2017) 5035-5043.

54] J. C. Dupré, P. Doumalin, Y. Belrhiti, I. Khlifi, O. Pop, M. Huger, Detection of cracks in refractory materials by an enhanced digital image correlation technique, Journal of Materials Science 53 (2) (2018) 977-993.

[55] I. Khlifi, O. Pop, J.-C. Dupré, P. Doumalin, M. Huger, Investigation of microstructure-property relantionships of magnesia-hercynite refractory composites by a refined digital image correlation technique, Journal of the European Ceramic Society 39 (13) (2019) 3893-3902.

[56] R. Vargas, J. Neggers, R. B. Canto, J. A. Rodrigues, F. Hild, Analysis of wedge splitting test on refractory castable via integrated DIC, Journal of the European Ceramic Society 36 (16) (2016) 4309-4317.

57] R. Vargas, J. Neggers, R. B. Canto, J. A. Rodrigues, F. Hild, Comparison of two full-field identification methods for the wedge splitting test on a refractory, Journal of the European Ceramic Society 38 (16) (2018) 5569 - 5579.

8] R. Vargas, J. Neggers, R. B. Canto, J. A. Rodrigues, F. Hild, Analysis of a castable refractory using the wedge splitting test and cohesive zone model, Journal of the European Ceramic Society 39 (13) (2019) 3903-3914.

[59] D. B. D. Stafuzza, J. A. Rodrigues, D. Miyaji, R. B. Canto, Application of the digital image correlation technique to the fracture energy method $\mathbf{5 3 3}$ for refractory concretes, in: Congresso Brasileiro de Engenharia e Ciência dos Materiais - 20 CBECiMat, Joinville, 2012, pp. 2300-2307, 534 (In Portuguese).

[60] D. Y. Miyaji, C. Z. Otofuji, M. D. Cabrelon, J. Medeiros, J. A. Rodrigues, The coke effect on the fracture energy of a refractory castable for the 536 petrochemical industry, in: Proceedings of the Unified International Technical Conference on Refractories (UNITECR 2013), Wiley Online 537 Library, 2014, pp. 1111-1116.

[61] A. H. A. Pereira, D. Y. Miyaji, M. D. Cabrelon, J. Medeiros, J. A. Rodrigues, A study about the contribution of the $\alpha$ - $\beta$ phase transition of 539 quartz to thermal cycle damage of a refractory used in fluidized catalytic cracking units, Cerâmica 60 (2014) 449-456.

[62] S. Ribeiro, C. C. D. Exposito, J. A. Rodrigues, Projeto, adaptação, instalação e testes preliminares para um sistema de medida de energia de fratura de materiais cerâmicos pelo método da cunha, Cerâmica 54 (2008) 418-426.

[63] J. Réthoré, S. Roux, F. Hild, An extended and integrated digital image correlation technique applied to the analysis fractured samples, Eur. J. 543 Comput. Mech. 18 (2009) 285-306.

[64] Z. Tomičević, F. Hild, S. Roux, Mechanics-aided digital image correlation, J. Strain Analysis 48 (2013) 330-343.

[65] R. Salomão, V. C. Pandolfelli, The role of hydraulic binders on magnesia containing refractory castables: calcium aluminate cement and hydratable alumina, Ceramics International 35 (8) (2009) 3117-3124.

6] V. Sciuti, F. Hild, V. Pandolfelli, T. Santos, B. Smaniotto, R. Canto, Digital Image Correlation applied to in situ evaluation of surface cracks upon curing of MgO-containing refractory castables, Journal of the European Ceramic Society (in press).

[67] V. Sciuti, R. Canto, J. Neggers, F. Hild, On the benefits of correcting brightness and contrast in global digital image correlation: Monitoring cracks during curing and drying of a refractory castable, Optics and Lasers in Engineering 136 (2020) 106316. 